\title{
Invariants of the Length Spectrum and Spectral Invariants of Planar Convex Domains
}

\author{
Georgi Popov* \\ Institute of Mathematics, Bulgarian Academy of Sciences, BG-Sofia 1090, Bulgaria and \\ Fachbereich Mathematik, Technische Hochschule Darmstadt, Schlossgartenstrasse 7, \\ D-64289 Darmstadt, Germany
}

Received: 21 January 1993/in revised form 8 June 1993

\begin{abstract}
This paper is concerned with a conjecture of Guillemin and Melrose that the length spectrum of a strictly convex bounded domain together with the spectra of the linear Poincare maps corresponding to the periodic broken geodesics in $\Omega$ determine uniquely the billiard ball map up to a symplectic conjugation. We consider continuous deformations of bounded domains $\Omega_{s}, s \in[0,1]$, with smooth boundaries and suppose that $\Omega_{0}$ is strictly convex and that the length spectrum does not change along the deformation. We prove that $\Omega_{s}$ is strictly convex for any $s$ along the deformation and that for different values of the parameter $s$ the corresponding billiard ball maps are symplectically equivalent to each other on the union of the invariant KAM circles. We prove as well that the KAM circles and the restriction of the billiard ball map on them are spectral invariants of the Laplacian with Dirichlet (Neumann) boundary conditions for suitable deformations of strictly convex domains.
\end{abstract}

\section{Introduction}

This paper is concerned with certain length spectrum invariants of a strictly convex and bounded planar domain $\Omega$ with a smooth boundary $\partial \Omega$. The motivation for studying such invariants comes from the inverse spectral problem formulated by Kac [12]. It is known $[10,18]$, that the length spectrum $\mathscr{C}(\Omega)$ of $\Omega$ is encoded in the spectrum of the Laplace operator $\Delta$ in $\Omega$ with Dirichlet (Neumann) boundary conditions, and that $\mathscr{S}(\Omega)$ can be extracted from the spectrum of $\Delta$ by means of the Poisson formula at least for generic domains. In this connection, Guillemin and Melrose [9] formulated the conjecture that the length spectrum of $\Omega$ and the spectra of the linear Poincare maps of the periodic broken geodesics of $\Omega$ form together a complete set of symplectic invariants for the corresponding billiard ball map $B$. As it was mentioned in [9], this conjecture seems to be a little optimistic and the local version of it is more hopeful.

The first result in this direction was obtained by Marvizi and Melrose [16] who described new length spectrum invariants of a strictly convex domain $\Omega$, studying the

\footnotetext{
* Supported by Alexander von Humboldt foundation
} 
asymptotics of the lengths of the closed broken geodesics approaching the boundary $\partial \Omega$. Let us take $l_{m n}$ arbitrarily in the set $\mathscr{L}(\Omega ; m, n)$ of lengths of all closed broken geodesics of $\Omega$ with $n$ vertices and winding number $m$. When $m$ is fixed and $n$ tends to infinity, $l_{m n}$ has an asymptotic expansion in powers of $n^{-2}$. The corresponding coefficients $c_{m k}, k=1,2, \ldots$, do not depend on the choice of $l_{m n}$ in $\mathscr{L}(\Omega ; m, n)$ and they are length spectrum as well as spectral invariants of $\Omega$ [16]. Colin de Verdière [4] proved that the labeled length spectrum and the spectra of the linear Poincare maps determine uniquely the Birkhoff invariants of a closed and elliptic broken ray in $\Omega \subset \mathbb{R}^{2}$. Recently this result was generalized in higher dimensions as well as for contact manifolds by Françoise and Guillemin [8].

De la Llave, Marco and Moriyón [15] proved that there are no non-trivial deformations of exact symplectic mappings $B_{s}, s \in[0,1]$, leaving the period spectrum fixed when $B_{s}$ are Anosov's mappings on a symplectic manifold. One of the reasons for symplectic rigidity in [15] is that all periodic points of $B_{s}$ are hyperbolic and form a dense set. Although the billiard ball map of a strictly convex domain is in the opposite situation, conjugation can still be made on a large part of the domain of $B_{s}$.

Consider the billiard ball map $B$ corresponding to a strictly convex domain $\Omega$ with a smooth boundary. $B$ is an exact symplectic map which is close to a completely integrable one near the boundary. Using that fact Lazutkin [14] proved that there exists a large family of invariant KAM circles $\Lambda(\omega)$ of $B$ with rotation numbers $\omega$ in a Cantor subset $\Theta$ of a positive Lebesgue measure in $[0, \varepsilon), \varepsilon>0$. The corresponding caustics $C(\omega)$ are strictly convex and smooth curves in $\Omega$ accumulating at $\partial \Omega$.

There are two invariants related to any invariant curve $\Lambda(\omega)$, namely the length $\ell(\omega)$ of the caustic $C(\omega)$ and the Lazutkin parameter $t(\omega)[1,14]$, (see also Sect. 2).

We prove in this paper that the vector function

$$
\Theta \ni \omega \rightarrow(\ell(\omega), t(\omega))
$$

is a length spectrum invariant for continuous deformations of the domain. The main result (see Theorem 2.1) says that the length spectrum determines uniquely (up to a symplectic conjugation) the invariant circles $\Lambda(\omega), \omega \in \Theta$, as well as the restriction of $B$ on them for continuous deformations of $\Omega$. We prove as well that the vector function (1.1), the invariant circles $\Lambda(\omega), \omega \in \Theta$, and the restriction of $B$ on them are spectral invariants of the Laplacian with Dirichlet (Neumann) boundary conditions for suitable continuous deformations of $\Omega$. The marked length spectrum of a bounded strictly convex domain is a map that assigns to any pair of positive integers $(m, n)$, $1 \leq m \leq n / 2$, the length of the longest (shortest) periodic broken geodesic in $\mathscr{L}(\Omega, m, n)$. Let $\Omega_{1}$ and $\Omega_{2}$ be two strictly convex and bounded domains with smooth boundaries. Suppose that the corresponding marked length spectra coincide. Then we show that the corresponding vector functions (1.1) coincide and the billiard ball maps are conjugated to each other on the union of the KAM invariant circles which improves Theorem 3 in [13].

The paper is organized as follows: In Sect. 2 we define the length spectrum $\mathscr{L}(\Omega)$ of a bounded domain with a smooth boundary and formulate the main result about the length spectrum invariants of continuous deformations of a strictly convex domain (see Theorem 2.1). Section 3 is devoted to a symplectic version of the KAM theorem for the billiard ball map which is the basic tool in the proof of the main results. First we introduce action-angle coordinates $(\theta, r) \in \mathbb{T} \times \mathbb{R}, \mathbb{T}=\mathbb{R} / 2 \pi \mathbb{Z}$, for the approximated interpolating Hamiltonian of $B$. In these coordinates $B$ is a small perturbation of the 
completely integrable map

$$
\mathbb{T} \times \Gamma \ni(\theta, r) \rightarrow\left(\theta+\left(\tau^{0}\right)^{\prime}(r), r\right), \quad \tau^{0}(r)=-\frac{4}{3} \zeta(r)^{3 / 2},
$$

where $\Gamma=(l-\varepsilon, l), 2 \pi l=\ell$ is the length of the boundary $\partial \Omega$, and $\zeta(l)=0$, the first derivative $\zeta^{\prime}(l)<0$, and $\zeta>0$ in $\Gamma$. Moreover, $\mathbb{T} \times\{l\}$ is a connected component of the boundary $\partial \Sigma$ of the phase space $\Sigma$ of $B$. We fix a Cantor subset $\Theta$ of rotation numbers determined via a small denominator condition which has a positive Lebesgue measure in the interval $[0, \varepsilon), \varepsilon>0$. Applying suitable KAM theorem we construct a symplectic normal form of $B$ on the union of the invariant circles of $B$ with rotation numbers in $\Theta$. In other words, we find symplectic coordinates $(\varphi, I) \in \mathbb{T} \times \mathbb{R}$ and smooth functions $K(I)$ and $Q(\varphi, I)$ in $\mathbb{R}$ and $\mathbb{T} \times \mathbb{R}$ respectively such that

$$
B(\varphi, I)=\left(\varphi+\tau^{\prime}(I), I\right)+Q(\varphi, I), \quad \tau(I)=-\frac{4}{3} K(I)^{3 / 2},
$$

in $\mathbb{T} \times \Gamma$, where $K(l)=0, K^{\prime}(l)<0$ and $K>0$ in $\Gamma$ while the vector function $Q$ has a zero of infinite order on $\mathbb{T} \times E$, and the Cantor set $E$ is defined by

$$
E=\left\{I \in \Gamma: \tau^{\prime}(I) / 2 \pi \in \Theta\right\} .
$$

Denote by $\mathscr{J}(\omega)$ the map inverse to the frequency mapping

$$
\Gamma \ni I \rightarrow \tau^{\prime}(I) / 2 \pi \text {. }
$$

The restriction of the functions $\mathscr{J}$ and $\tau \circ \mathscr{J}$ on $\Theta$ is a symplectic invariant of $B$ and it has a simple geometric meaning. We prove in Sect. 4 that $\mathscr{J}(\omega)$ is equal to $\ell(\omega) / 2 \pi$ while $\tau(\mathscr{J}(\omega))=-t(\omega)$ for any $\omega \in \Theta$. In particular, the Legendre transform

$$
\mathscr{T}(\omega)=\omega \mathscr{Z}(\omega)-\tau(\mathscr{J}(\omega)) / 2 \pi
$$

of $\tau(I) / 2 \pi$ is given on $\Theta$ by

$$
2 \pi \mathscr{T}(\omega)=\omega \ell(\omega)+t(\omega), \quad \forall \omega \in \Theta .
$$

In Sect. 5 we complete the proof of Theorem 2.1. The main ideas here are:

1. Let $[0, b] \ni s \rightarrow \Omega_{s}, b>0$, be a continuous deformation of strictly convex bounded domains with smooth boundaries. Following an argument due to Birkhoff, we prove that for any pair $(m, n) \in \mathbb{Z}_{+}^{2}, 1 \leq m \leq n / 2$, the function

$$
[0, b] \ni s \rightarrow T_{m n}(s)=\max \left\{t: t \in \mathscr{L}\left(\Omega_{s}, m, n\right)\right\},
$$

is continuous, and that $\mathscr{L}\left(\Omega_{s}\right)$ is a subset of $\mathbb{R}$ of Lebesgue measure zero for any $s$. If the length spectrum of $\Omega_{s}$ is independent of $s$ along the deformation, the continuous function $T_{m n}(s)$ takes values in the set $\mathscr{L}\left(\Omega_{s}\right)=\mathscr{L}\left(\Omega_{0}\right)$ which does not contain intervals. Hence,

$$
T_{m n}(s)=T_{m n}(0), \quad s \in[0, b]
$$

In other words, if the length spectrum of a strictly convex domain remains constant along a small continuous deformation of the boundary then so does the marked length spectrum. In particular, the results in [4] hold for such deformations.

2. Fix $\omega \in \Theta$ and choose a sequence $\left(m_{j}, n_{\jmath}\right) \in \mathbb{Z}_{+}^{2}, j=1,2, \ldots$, such that

$$
\left|m_{j} / n_{j}-\omega\right| \leq n_{j}^{-1 / 2}
$$

We prove that

$$
2 \pi \mathscr{T}(\omega)=\lim _{j \rightarrow \infty}\left(T_{m_{j} n_{\jmath}}(s) / n_{\jmath}\right), \quad \forall s \in[0, b],
$$


and taking into account (1.3) we obtain

$$
\mathscr{T}_{s}(\omega)=\mathscr{T}_{0}(\omega), \quad \forall \omega \in \Theta, \quad \forall s \in[0, b] .
$$

Since $\Theta$ has no isolated points, differentiating the last equality with respect to $\omega$ we obtain

$$
\ell_{s}(\omega)=\ell_{0}(\omega), \quad t_{s}(\omega)=t_{0}(\omega), \quad \forall \omega \in \Theta, \quad \forall s \in[0, b] .
$$

A close idea has been used in [21] to study the invariants of the period spectrum of an elliptic periodic trajectory of a contact manifold.

Equality (1.4) is a consequence of the following important estimate (see Theorem 5.1)

$$
\left|l_{m n}-2 \pi n \mathscr{T}(m / n)\right| \leq C_{p} n^{-p}, \quad \forall p>0,
$$

which holds for any pair $(m, n) \in \mathbb{Z}_{+}^{2}, 1 \leq m \leq n / 2$, satisfying the inequality

$$
\operatorname{dist}(m / n, \Theta) \leq n^{-1 / 2},
$$

and for any $l_{m n}$ arbitrary chosen in $\mathscr{L}(\Omega, m, n)$. Here, for each $p>0, C_{p}$ is a positive constant which depends neither on $m$ and $n$ nor on the choice of $l_{m n}$.

The proof of (1.6) is based on the KAM theorem and on a Birkhoff-Lewis type theorem. An alternative and simpler proof of (1.4) can be obtained following the proof of Theorem 3 in [13]. One can consider (1.6) as a nontrivial generalization of Theorem 5.15, [16]. Indeed, since $0 \in \Theta$, if we fix $m$, let $n$ go to infinity, and expand $\mathscr{T}(t)$ in Taylor series at $t=0$, we obtain the result in [16] mentioned above as a consequence of (1.6) (see Corollary 5.1). The invariants $c_{m k}$ of Marvizi and Melrose are explicitly given by the Taylor coefficients of $\mathscr{T}(t)$ at $t=0$. We use essentially (1.6) and its proof when studying the spectral invariants of the Laplace operator in $\Omega$. Note that instead of $1 / 2$ in the exponent in the right-hand-side of (1.7) one can take any $0<\varepsilon<1$.

3. We assume that $\Omega_{s}$ is strictly convex only in a small interval $\left[0, b_{0}\right), b_{0}>0$. To show that $\Omega_{s}$ is strictly convex for any $s$ in $[0,1]$ along the deformation, we use (1.5) as well as the integral invariants $I^{(k+1)}(0)$ of Marvizi and Melrose [16] which are integrals of polynomials of the curvature of $\partial \Omega$ and its derivatives. In particular we prove that $I^{(k+1)}(0)=2 \pi \mathscr{R}^{(k)}(0), k=1,2, \ldots$, where $\mathscr{B}(t)$ is the function inverse to $t=K(I)$ and $\mathscr{R}^{(k)}(0)$ are the corresponding derivatives at $t=0$.

Section 6 is devoted to spectral invariants of the Laplacian $\Delta=-\partial^{2} / \partial x_{1}^{2}-\partial^{2} / \partial x_{2}^{2}$ in $\Omega$ with Dirichlet (Neumann) boundary conditions. We suppose that $\Omega$ is a strictly convex bounded domain in $\mathbb{R}^{2}$ with a smooth boundary. Then, the spectrum of $\Delta$ consists of non-negative eigenvalues tending to infinity. Guillemin and Melrose formulated in [9] the conjecture that the spectrum of the Laplace operator in $\Omega$ with Dirichlet (Neumann) boundary conditions determines uniquely the billiard ball map. Partial affirmative answer to this conjecture is given in Sect. 6 (see Theorem 6.2). We prove that the vector function (1.1), the invariant circles $\Lambda(\omega), \omega \in \Theta$, as well as the restriction of the billiard ball map on them are spectral invariants of the Laplacian for suitable continuous deformations of a strictly convex domain. In particular, the corresponding billiard ball maps are conjugated to each other on a large subset of $\Sigma$ of a positive Lebesgue measure. We investigate the singularities of the distribution

$$
Z(t)=\operatorname{trace} \cos \left(t \Delta^{1 / 2}\right)=(1 / 2) \sum \exp (i \lambda t)
$$


where the sum is taken over all $\lambda$ with $\lambda^{2}$ in the spectrum of $\Delta$ counted with multiplicity. The singular support of $Z(t)$ satisfies the Poisson relation

$$
\text { sing. supp. } Z(t) \subseteq\{T \in \mathbb{R}: \pm T \in \mathscr{L}(\Omega)\} \cup\{0\}
$$

$[2,18]$. The inverse relation may not always be true, because singularities created by different closed broken geodesics may cancel each other. It is known that (1.9) turns into equality in the generic case when all periodic broken geodesics are nondegenerate and of different lengths [18].

Let $(m, n) \in \mathbb{Z}_{+}^{2}$ satisfy (1.7). In Sect. 6 we prove under the natural condition (6.1) that

$$
T_{m n} \in \text { sing. supp. } Z
$$

if $n \geq n_{0}(\Omega)$. Hence, a large part of the marked length spectrum is encoded in the spectrum of the Laplacian for such domains. For $m=1$ and $n$ sufficiently large this result has been proved in [16]. The main idea in [16] is to write $Z(t)$ in a neighborhood of $T_{1 n}$ as a Lagrangian distribution with a suitable phase function and then to apply a result of Soga. We use another representation on $Z(t)$ in a neighborhood of $T_{m n}$ which is based on the KAM theorem and the results obtained in Sect. 5.

\section{Length Spectrum}

Let $\Omega$ be a bounded domain in $\mathbb{R}^{2}$ with a smooth boundary $\partial \Omega$. The length spectrum $\mathscr{L}(\Omega)$ of $\Omega$ is defined as the set of lengths of all periodic generalized geodesics $\widetilde{\gamma}$ of $\bar{\Omega}(\widetilde{\gamma}$ is the projection on $\bar{\Omega}$ of a closed generalized bicharacteristic of the Hamiltonian $p(x, \xi)=|\xi|^{2}-1$, (cf. [11], Def. 24.3.7)). By definition, if $\widetilde{\gamma}$ is a primitive generalized geodesic of $\bar{\Omega}$ of length $L(\widetilde{\gamma})$ then $n L(\widetilde{\gamma})$ belongs to the length spectrum of $\Omega$ for any $n$-positive and integer. Suppose in addition that $\Omega$ is strictly convex. Then any generalized bicharaceristic of $\Omega$ is either a broken bicharacteristic reflecting at the boundary by the usual law of the geometric optics or it is a gliding ray traveling along the boundary. Hence, $\mathscr{L}(\Omega)=\mathscr{L}_{b}(\Omega) \cup \mathscr{L}(\partial \Omega)$ in this case where $\mathscr{L}_{b}(\Omega)$ is the set of lengths of all closed broken geodesics and $\mathscr{L}(\partial \Omega)=\{n \ell: n \in \mathbb{N}\}, \ell$ being the length of the boundary.

The broken bicharacteristic flow induces a discrete dynamical system on the boundary

$$
B: \Sigma \rightarrow \Sigma, \quad \Sigma=\left\{(x, \xi) \in T^{*} \partial \Omega:|\xi| \leq 1\right\},
$$

called billiard ball map which is defined as follows: Pick $\varrho=(x, \xi)$ in $T^{*} \partial \Omega$ with $|\xi|<1$ and set $\varrho^{ \pm}=\pi^{ \pm}(\varrho)=\left(x, e^{ \pm}(\varrho)\right)$. Here $e^{ \pm}(\varrho) \in\left(\mathbb{R}^{2}\right)^{*}$ are unit convectors such that

$$
\pm\left\langle e^{ \pm}(x, \xi), n(x)\right\rangle>0, \quad\left\langle e^{ \pm}(x, \xi), v\right\rangle=\langle\xi, v\rangle, \quad \forall v \in T_{x} \partial \Omega,
$$

$n(x)$ being the inward normal to $\partial \Omega$ at $x$. Via the canonical inner product in $\mathbb{R}^{2}$ we identify $e^{ \pm}(\varrho)$ with a vector $\widetilde{e}^{ \pm}(\varrho)$ in $\mathbb{R}^{2}$. The bicharacteristic

$$
\mathbb{R} \ni t \rightarrow\left(x+t \widetilde{e}^{+}, e^{+}\right)
$$

of $S^{*} \mathbb{R}^{2}=\left\{(y, \eta) \in T^{*} \mathbb{R}^{2}:|\eta|=1\right\}$ passing through $\pi^{+}(\varrho)$ intersects $\left(T^{*} \mathbb{R}^{2}\right)_{\mid \partial \Omega}$ at a second point $\left(y, e^{+}\right)$. Define $\eta \in T_{y}^{*} \partial \Omega$ by the equality

$$
\left\langle e^{+}, v\right\rangle=\langle\eta, v\rangle, \quad \forall v \in T_{y} \partial \Omega .
$$


Then $|\eta|<1$ and $B$ sends $(x, \xi)$ to $(y, \eta)$. Moreover, any point in $\partial \Sigma$ is a fixed point of $B$.

Defined in this way the billiard ball map is exact symplectic in the interior of $\Sigma$, indeed

$$
B^{*} \sigma-\sigma=d T
$$

where $\sigma$ is the canonical one-form in $T^{*} \partial \Omega$ and $T(x, y)=|x-y|$ is the distance between $x$ and $y$ in $\mathbb{R}^{2}, y$ being the first component of $B(x, \xi)=(y, \eta)$ (see Proposition 2.3 in [9]).

Near the boundary of $\Sigma$ the billiard ball map $B$ is a small perturbation of a completely integrable map for which the KAM theorem can be applied [17, 14]. In particular, there exists a large family of invariant circles $\Lambda(\omega)$ of $B$ enumerated by their rotation numbers $\omega \in \Theta$, where $\Theta$ is a Cantor subset of the interval $(0,1 / 2]$ with a positive measure (see Sect. 3). For each $\omega \in \Theta$, denote by $C(\omega)$ the corresponding caustic in $\Omega$, i.e. the envelope of the rays $\left\{x+t \widetilde{e}^{+}(x, \xi): t>0\right\},(x, \xi) \in \Lambda(\omega)$, issuing from $\Lambda(\omega)$. Then $C(\omega)$ is a smooth and strictly convex curve in $\Omega$ and the boundary $\partial \Omega$ is an evolute of $C(\omega)$ [14]. In other words, if we loop a string with a suitable length $T(\omega)$ around $C(\omega)$, lean a pen against it and draw, we get $\partial \Omega$. The Lazutkin parameter of $C(\omega)$ is defined by

$$
t(\omega)=T(\omega)-\ell(\omega)
$$

where $\ell(\omega)$ is the length of $C(\omega)$.

We consider a continuous deformation

$$
[0,1] \ni s \rightarrow \Omega_{s} \subset \mathbb{R}^{2}
$$

of bounded domains with smooth boundaries $\partial \Omega_{s}=\left\{x^{s}(t): t \in \mathbb{T}\right\}$, such that the mapping $[0,1] \ni s \rightarrow x^{s}(\cdot) \in C^{\infty}\left(\mathbb{T}, \mathbb{R}^{2}\right)$ is continuous. For any strictly convex domain $\Omega_{s}$ we denote by $B_{s}, \Lambda_{s}(\omega)$ and $C_{s}(\omega)$ the corresponding billiard ball map, invariant circle and caustic with a rotation number $\omega$. Consider the Cantor set $\Theta$ defined by (3.9). According to (3.19), the union $\Lambda_{s}$ of the invariant circles $\Lambda_{s}(\omega)$, $\omega \in \Theta$, is a set of positive Lebesgue measure in $T^{*} \partial \Omega_{s}$. The main result in this paper is:

Theorem 2.1. Let $[0,1] \in s \rightarrow \Omega_{s}$ be a continuous deformation of bounded domains in $\mathbb{R}^{2}$ with smooth boundaries. Suppose that $\Omega_{0}$ is strictly convex and

$$
\mathscr{L}\left(\Omega_{s}\right)=\mathscr{L}\left(\Omega_{0}\right), \quad s \in[0,1] .
$$

Then:

(i) $\Omega_{s}$ is strictly convex for any $s \in[0,1]$,

(ii) there exists a continuous family of smooth exact symplectic mappings

$$
\chi_{s}: T^{*} \partial \Omega_{0} \rightarrow T^{*} \partial \Omega_{s},
$$

such that

$$
\begin{gathered}
\chi_{s}\left(\Lambda_{0}(\omega)\right)=\Lambda_{s}(\omega), \quad \forall \omega \in \Theta \\
\chi_{s} \circ B_{0}=B_{s} \circ \chi_{s} \quad \text { on } \Lambda_{0}
\end{gathered}
$$

for any $s \in[0,1]$,

(iii) for any $\omega \in \Theta$ there exists a continuous family of caustics $[0,1] \rightarrow C_{s}(\omega)$ in $\Omega_{s}$ and

$$
\ell_{s}(\omega)=\ell_{0}(\omega), \quad t_{s}(\omega)=t_{0}(\omega), \quad s \in[0,1]
$$




\section{KAM Theorem}

In this section we formulate a symplectic version of the KAM theorem for a family of exact symplectic mappings depending continuously on a parameter which will be the basic technical tool in the proof of the main results. This theorem is close to Theorem 1.1 in [21]. First we consider a continuous deformation of bounded strictly convex domains $\Omega_{s}, s \in[0, b], b>0$, in $\mathbb{R}^{2}$, with smooth boundaries $\partial \Omega_{s}$ of length $\ell_{s}$, and introduce action-angle coordinates for the approximated interpolating Hamiltonians of the corresponding billiard ball maps $B_{s}$.

Performing a suitable change of the variables in $\mathbb{R}^{2}$, we consider $\Omega_{s}, s \in[0, b]$, as a Riemannian manifold with a base $\Omega=\Omega_{0}$ and metric $g_{s}$ depending continuously on $s$. The boundary $\partial \Omega_{s}$ is given by $\partial \Omega$ equipped with the induced metric $g_{s}^{0}$. The corresponding billiard ball map $B_{s}$ is defined in the same manner as in Sect. 2. Its phase space coincides with the coball bundle

$$
\Sigma_{s}=\left\{(x, \xi) \in T^{*} \partial \Omega: g_{s}^{0}(x, \xi) \leq 1\right\} .
$$

Let us denote by $\partial \Sigma_{s}^{+}$one of the two components of the boundary of $\Sigma_{s}$. Since $\partial \Omega_{s}$ is strictly geodesically convex, $B_{s}$ can be written as a small perturbation of a completely integrable map as follows (see [16]): there exists a smooth function $\zeta_{s}$ called an approximated interpolating Hamiltonian which defines $\partial \Sigma_{s}^{+}\left(\zeta_{s}=0\right.$ and $\nabla \zeta_{s} \neq 0$ on $\left.\partial \Sigma_{s}\right), \zeta_{s} \geq 0$ on $\Sigma_{s}$, and such that in any local coordinates $\varrho=(x, \xi)$ in a local chart $U$ in $T^{*} \partial \Omega$ we have

$$
\begin{aligned}
& B_{s}(\varrho)=\exp \left(-2 \zeta_{s}(\varrho)^{1 / 2} H_{\zeta_{s}}\right)(\varrho)+R_{s}(\varrho), \quad \varrho \in \Sigma_{s} \cap U, \\
& R_{s}(\varrho)=O\left(\zeta_{s}^{\infty}(\varrho)\right) \text { at } \partial \Sigma_{s}^{+} \cap U .
\end{aligned}
$$

Here $t \rightarrow \exp \left(t H_{\zeta_{s}}\right)(\varrho)$ stands for the integral curve of the Hamiltonian vector field $H_{\zeta_{s}}$ starting at $\varrho \in \Sigma_{s}, R_{s}$ is a continuous family of smooth functions in $U$, and (3.2) means that

$$
\left|\partial_{x}^{\alpha} \partial_{\xi}^{\beta} R_{s}(x, \xi)\right| \leq C_{N \alpha \beta} \zeta_{s}(x, \xi)^{N}, \quad(x, \xi) \in U,
$$

for any indices $\alpha, \beta, N$. Moreover, the mapping $[0, b] \ni s \rightarrow \zeta_{s}(\cdot) \in C^{\infty}\left(T^{*} \partial \Omega\right)$ is continuous.

We are going to describe action-angle coordinates for the Hamiltonian $\zeta_{s}$. To simplify the notations we drop the index $s$. Denote by $M_{r}$ the closed curve $\{\zeta=r\}$ in $T^{*} \partial \Omega$, where $r$ varies in a small neighborhood of the origin. For any $\varrho \in M_{r}$ consider the map $\mathbb{R} \ni t \rightarrow \exp \left(t H_{\zeta}\right)(\varrho) \in M_{r}$ and denote by $2 \pi \Pi(r)$ its period. Let $S$ be a section transversal to $M_{0}$ in $\Sigma$. It is equipped with local coordinates $S \ni \varrho \rightarrow \zeta(\varrho)$. Denote by $\bigcirc$ the discrete group in $\mathbb{R} \times S$ generated by

$$
\mathbb{R} \times S \ni(t, \zeta(\varrho)) \rightarrow(t+2 \pi \Pi(\zeta(\varrho)), \zeta(\varrho)) .
$$

Let $(\mathbb{R} \times S) / \bigcirc$ be the corresponding factor space. It is a symplectic manifold, $d \zeta \wedge d t$ is a symplectic two-form on it, and the mapping

$$
\mathbb{R} \times S \ni(t, \varrho) \rightarrow \exp \left(t H_{\zeta}\right)(\varrho) \in T^{*} \partial \Omega
$$

lifts to a symplectic diffeomorphism from $(\mathbb{R} \times S) / \bigcirc$ to a neighborhood of $M_{0}$. Making suitable symplectic change of the variables in $\mathbb{R} \times S$,

$$
\theta=t / \Pi(\zeta), \quad r=g(\zeta)
$$


we can suppose that $(\mathcal{O}$ is generated by $(\theta, r) \rightarrow(\theta+2 \pi, r)$ while the symplectic two-form becomes $d \theta \wedge d r$. It is easy to see that the first derivative $g^{\prime}(\zeta)=-\Pi(\zeta)$, which yields

$$
r(\zeta)=-\int_{0}^{\zeta} \Pi(t) d t+l, \quad l=l(0)=\ell / 2 \pi .
$$

Denote by $\zeta(r)$ the function inverse to $r(\zeta)$. We have obtained symplectic coordinates $\left(\theta_{s}(x, \xi), r_{s}(x, \xi)\right)$ in a neighborhood of $\partial \Sigma_{s}^{+}$in $T^{*} \partial \Omega$ with values in $\mathbb{T} \times \mathbb{R}$ such that $\partial \Sigma_{s}^{+}=\left\{r_{s}=l_{s}\right\}, l_{s}=\ell_{s} / 2 \pi$ and $\Sigma_{s} \subset\left\{r_{s} \leq l_{s}\right\}$. Fix $\varepsilon>0$ and set $\Gamma_{s}=\left(l_{s}-\varepsilon, l_{s}\right), \mathbb{A}_{s}=\mathbb{T} \times \Gamma_{s}$.

The exact symplectic map $B_{s}$ is generated in these coordinates by the function

$$
G_{s}(\theta, r)=-\frac{4}{3} \zeta_{s}(r)^{3 / 2}+Q_{s}(\theta, r), \quad(\theta, r) \in \mathbb{A}_{s},
$$

where

$$
\zeta_{s}\left(l_{s}\right)=0, \quad \zeta_{s}^{\prime}\left(l_{s}\right)=-1 / \Pi_{s}(0)<0, \quad \partial_{\theta}^{\alpha} \partial_{r}^{\beta} Q_{s}\left(\theta, l_{s}\right)=0, \quad \forall \theta \in \mathbb{T},
$$

for any indices $\alpha \geq 0, \beta \geq 0$, and $s \in[0, b]$. Hereafter we say that $G_{s}$ generates the exact symplectic map $B_{s}$ in $\mathbb{A}_{s}$ if

$$
\operatorname{graph}\left(B_{s}\right)=\left\{\left(B_{s}(x, \xi),(x, \xi)\right):(x, \xi) \in \mathbb{A}_{s}\right\}
$$

is parameterized by

$$
\operatorname{graph}\left(B_{s}\right)=\left\{\left(\theta, r-\frac{\partial G_{s}}{\partial \theta}(\theta, r) ; \theta-\frac{\partial G_{s}}{\partial r}(\theta, r), r\right):(\theta, r) \in \mathbb{A}_{s}\right\}
$$

where

$$
\left|\frac{\partial^{2} G_{s}}{\partial \theta \partial r}(\theta, r)\right|<1, \quad \forall(\theta, r) \in \mathbb{A}_{s} .
$$

Multiplying $G_{s}$ by a cut-off function we can suppose that it is equal to zero for $r \leq l_{s}-2 \varepsilon / 3$. From now on we denote by $B_{s}$ the corresponding modified exact symplectic mappings. Note that $B_{s}, \zeta_{s}$ as well as the exact symplectic mappings $\psi_{s}^{0}$ defined by

$$
\left(\psi_{s}^{0}\right)^{-1}(x, \xi)=\left(\theta_{s}(x, \xi), r_{s}(x, \xi)\right)
$$

depend continuously on $s$ in the corresponding $C^{\infty}$ semi-norms.

The billiard ball map $B_{s}$ is a small perturbation of the completely integrable mapping generated in $\mathbb{A}_{\mathrm{s}}$ by $\tau_{s}^{0}(r)=-\frac{4}{3} \zeta_{s}(r)^{3 / 2}$. In what follows we apply a symplectic version of the KAM theorem to $B_{s}$ which is close to Theorem 1.1 in [21]. In contrast to [21], the generating function $\tau_{s}^{0}(r)$ has singularity at $r=l_{s}$.

As a consequence of (2.3) and Lemma 5.2 we easily obtain $\ell_{s}=\ell_{0}$ in $[0, b]$. Indeed, the continuous function $[0, b] \ni s \rightarrow \ell_{s}$ takes values in the set $\mathscr{L}\left(\Omega_{s}\right)=\mathscr{L}\left(\Omega_{0}\right)$ which does not contain intervals, according to Lemma 5.2. Hence, $\ell_{s}$ does not depend on $s$. To simplify the notations we set $\Gamma=\Gamma_{0}$ and $\mathbb{A}=\mathbb{A}_{0}$.

We are going to define the Cantor set $\Theta$. Fix $\sigma>1, \mu>0$, and for any $a>0$ and $N \in \mathbb{Z}_{+}$define the Cantor set $\Theta(a, \mu, N)$ by the small denominator condition as follows:

$$
\Theta(a, \mu, N)=\left\{\omega \in \mathbb{R}:\left|\omega k_{1}-k_{2}\right| \geq \mu a^{N}|k|^{-\sigma} \text { for any } k=\left(k_{1}, k_{2}\right) \in \mathbb{Z}^{2} \backslash\{0\}\right\} .
$$


Fix $0<C<1$ and $0<a_{0}<0$, and denote

$$
\Theta^{*}(a, \mu, N)=\Theta(a, \mu, N) \cap\left[C a, C^{-1} a\right], \quad 0<a<a_{0} .
$$

Consider the Cantor subset of $[0,1 / 2)$ defined by

$$
\Theta=\cup\left\{\Theta^{*}(a, \mu, N): 0<a \leq \varepsilon(\mu, N), N \in \mathbb{Z}_{+}\right\} \cup\{0\},
$$

where

$$
\varepsilon(\mu, N)=\varepsilon_{N} \mu^{M}, \quad M=N+2,
$$

while the positive constants $\varepsilon_{N}$ will be specified later.

This set is of a positive Lebesgue measure in $\mathbb{R}$ and even

$$
\varepsilon-\operatorname{meas}(\Theta \cap[0, \varepsilon)) \leq C_{p} \varepsilon^{p}, \quad 0<\varepsilon<\varepsilon_{0}
$$

where $C_{p}$ is a positive constant for any $p \geq 1$. The following theorem provides a symplectic normal form for the family of symplectic mappings $B_{s}$ in a neighborhood of $\partial \Sigma_{s}^{+}$. As above we assume that $B_{s}$ is generated by a function $G_{s}$ in $\mathbb{A}$ and that $B_{s}$ coincides with the identity mapping in $\mathbb{T} \times\left[l_{0}-\varepsilon, l_{0}-2 \varepsilon / 3\right]$.

Theorem 3.1. Let $[0, b] \ni s \rightarrow B_{s} \in C^{\infty}(\mathbb{A}, \mathbb{A})$ be a continuous deformation of exact symplectic mappings. Suppose that the corresponding generating functions $G_{s}$ satisfy (3.5) and (3.6), and $\ell_{s}=\ell_{0}$ for any s. Then there is a Cantor set $\Theta$ defined by (3.9) with suitable $\varepsilon_{N}>0$ and there exist continuous in $[0, b]$ families of exact symplectic mappings $\psi_{s} \in C^{\infty}(\mathbb{T} \times \mathbb{R}, \mathbb{T} \times \mathbb{R})$ and functions $K_{s} \in C^{\infty}(\mathbb{R}), Q_{s}^{0} \in C^{\infty}(\mathbb{T} \times \mathbb{R})$ such that:

(i) $K_{s}\left(l_{0}\right)=0, K_{s}^{\prime}\left(l_{0}\right)<0, K_{s}(t)>0$ in $\Gamma$, and the exact symplectic map $B_{s}^{0}=\psi_{s}^{-1} \circ B \circ \psi_{s}$ is generated in $\mathbb{A}$ by

$$
G_{s}^{0}(\varphi, I)=\tau_{s}(I)+Q_{s}^{0}(\varphi, I), \quad \tau_{s}(I)=-\frac{4}{3} K_{s}(I)^{3 / 2}, \quad(\varphi, I) \in \mathbb{A},
$$

where

$$
Q_{s}^{0}(\varphi, I)=0 \quad \text { on } \quad \mathbb{T} \times E_{s}
$$

and $E_{s}=\left\{I \in \Gamma: \tau_{s}^{\prime}(I) / 2 \pi \in \Theta\right\}$.

(ii) $K_{s}, Q_{s}^{0}$, and the generating function $S_{s}(\theta, I)$ of $\psi_{s}$ satisfy the estimates

$$
\begin{aligned}
\left|D_{I}^{\alpha}\left(K_{s}(I)-\zeta_{s}(I)\right)\right|+\left|D_{I}^{\alpha} D_{\varphi}^{\beta} Q_{s}^{0}(\varphi, I)\right| & \leq C_{\alpha \beta p}\left|l_{0}-I\right|^{p} \\
\left|D_{I}^{\alpha} D_{\theta}^{\beta} S_{s}(\theta, I)\right| & \leq C_{\alpha \beta p}\left|l_{0}-I\right|^{p}
\end{aligned}
$$

in $\mathbb{T} \times\left[l_{0}-\varepsilon_{0}, l_{0}+\varepsilon_{0}\right], \varepsilon_{0}>0$, for any $s \in[0, b]$ and any indices $\alpha \geq 0, \beta \geq 0$ and $p>0$. 
The proof of Theorem 3.1 is given in the Appendix. First we construct exact symplectic mappings conjugating the billiard ball maps $B_{s}$ in suitable domains away from the singularity set $\left\{r=l_{0}\right\}$ of $B_{s}$ and then we patch them together using the uniqueness of the KAM circle with a given rotation number. Proposition A.1 plays an important role here.

In view of (3.6), (3.13), and the equality $\ell_{s}=\ell_{0}$, the frequency map

$$
\Gamma \ni I \rightarrow \omega=\tau_{s}^{\prime}(I) / 2 \pi \in\left(0, \omega_{0}\right), \quad \omega_{0}>a_{0}
$$

is invertible if $\Gamma$ is sufficiently small. Denote by $\mathscr{J}_{s}(\omega)$ the inverse map to (3.15) in $\left[0, \omega_{0}\right)$. Then $E_{s}=\mathscr{Z}_{s}(\Theta)$ and we have

$$
\mathscr{Z}_{s}(\omega)=l_{0}-c_{s} \omega^{2}+O\left(\omega^{4}\right), \quad l_{0}=\ell_{0} / 2 \pi, \quad c_{s}=\pi^{2} \Pi_{s}(0)^{3}>0
$$

Moreover, $\mathscr{J}_{s}(\omega)$ can be extended to a smooth even function in $\mathbb{R}$. Set $\chi_{s}=\psi_{s}^{0} \circ \psi_{s}$, where $\psi_{s}^{0}$ is defined by (3.7). Since $E_{s}$ has no isolated points, (3.12) means that $Q_{s}^{0}$ has a zero of infinite order at $\mathbb{T} \times E_{s}$. In particular,

$$
B_{s}^{0}(\varphi, I)=\left(\varphi+\tau_{s}^{\prime}(I), I\right), \quad(\varphi, I) \in \mathbb{T} \times E_{s},
$$

and

$$
[0, b] \ni s \rightarrow \Lambda_{s}(\omega)=\chi_{s}\left(\mathbb{T} \times\left\{\mathscr{J}_{s}(\omega)\right\}\right)
$$

is a continuous family of invariant circles of $B_{s}$ with a rotation number $\omega \in \Theta$ which accumulate at $\partial \Sigma_{s}^{+}=\chi_{s}\left(\mathbb{T} \times\left\{l_{0}\right\}\right)$ since $\mathscr{J}_{s}(0)=l_{0}$. Denote by $\Lambda_{s}$ the union of the invariant circles $\Lambda_{s}(\omega), \omega \in \Theta$, and consider the function

$$
h_{s}(x, \xi)=K_{s}\left(\chi_{s}^{-1}(x, \xi)\right)
$$

where $K_{s}$ is introduced by Theorem 3.1. Since $Q_{s}^{0}$ has a zero of infinite order at $E_{s}$ and $l_{0} \in E_{s}, h_{s}$ is an approximated interpolating Hamiltonian of $B_{s}$. Thus we obtain

Corollary 3.1. We have

$$
B_{s}(\varrho)=\exp \left(-2 h_{s}(\varrho)^{1 / 2} H_{h_{s}}\right)(\varrho)+R_{s}(\varrho), \quad \varrho \in \Sigma_{s} \cap U
$$

in any local coordinates in a chart $U$ in $T^{*} \partial \Omega$ where $R_{s}(\varrho) \in C^{\infty}(U)$ is continuous with respect to $s \in[0, b]$ and $R_{s}$ has a zero of infinite order at $\Lambda_{s} \cap U$.

We are going to show in Proposition 4.1 that $h_{s}(\varrho)$ coincides with $\left(3 t_{s}(\omega) / 4\right)^{2 / 3}$ for any $\varrho \in \Lambda_{s}(\omega)$ and $\omega \in \Theta$, where $t_{s}(\omega)$ is the Lazutkin parameter of the invariant circle $\Lambda_{s}(\omega)$. Note that, according to (3.10),

$$
\operatorname{meas}(U)-\operatorname{meas}\left(U \cap \Lambda_{s}\right) \leq C_{N}(\operatorname{meas}(U))^{N}, \quad s \in[0, b]
$$

for any sufficiently small neighborhood $U$ of $\partial \Sigma_{s}^{+}$. 


\section{Caustics and Lazutkin's Parameter}

This section is devoted to the geometry of the caustics of a strictly convex domain. Our aim is to give a simple geometric interpretation of the function

$$
\Theta \ni \omega \rightarrow\left(\mathscr{J}_{s}(\omega), K_{s}\left(\mathscr{J}_{s}(\omega)\right)\right.
$$

where $K_{s}$ is introduced by Theorem 3.1 and $\mathscr{J}_{s}$ is the function inverse to the frequency map defined above. To simplify the notations we drop the index $s$.

Fix $\omega \in \Theta$ and consider the invariant circle $\Lambda(\omega)$ of $B$ and the corresponding caustic $C(\omega)$ with a rotation number $\omega$. Take $\varrho=(x, \xi)$ arbitrarily in $\Lambda(\omega)$. The projections $t \rightarrow x+t \widetilde{e}^{ \pm}(\varrho)$ of the bicharacteristics of $S^{*} \mathbb{R}^{2}=\left\{(y, \eta) \in T^{*} \mathbb{R}^{2}\right.$ : $|\eta|=1\}$ passing through $\pi^{ \pm}(\varrho)$ are tangent to the caustic $C(\omega)$ which is smooth and strictly convex (see [14]). Let $y^{ \pm}=y^{ \pm}(\varrho)$ be the corresponding points of tangency. Denote by $\left|x y^{ \pm}\right|$the distance between $x$ and $y^{ \pm}$and by $\left|y^{-} \frown y^{+}\right|$the length of the shortest arc in $C(\omega)$ connecting $y^{-}$with $y^{+}$. The Lazutkin parameter of the caustic $C(\omega)$ is given by

$$
t(\omega)=\left|x y^{-}\right|+\left|x y^{+}\right|-\left|y^{-} \frown y^{+}\right|
$$

and it does not depend on the choice of $x \in \partial \Omega$ (see $[14,1]$ ). As above denote by $\ell(\omega)$ the length of the caustic $C(\omega)$. The main result in this section is:

Proposition 4.1. For any $\omega \in \Theta$ we have

$$
\mathscr{J}(\omega)=\ell(\omega) / 2 \pi, \quad \tau(\mathscr{J}(\omega))=-t(\omega) .
$$

Proof. Consider the flow-out

$$
\mathscr{M}(\omega)=\left\{\exp \left(t H_{g}\right)\left(\pi^{+}(\varrho)\right): \varrho \in \Lambda(\omega), 0 \leq t \leq q(\varrho)\right\}
$$

of $\Lambda(\omega)$ with respect to the Hamiltonian $g(y, \eta)=|\eta|-1,(y, \eta) \in T^{*} \mathbb{R}^{2}$, where $q(\varrho)$ is the time $t$ for which a point starting at $x$ and travelling with unit speed along the ray $\left.t \rightarrow x+t \widetilde{e}^{+}(\varrho)\right), t \geq 0$, reaches $y^{+} \in C(\omega)$. Then $\mathscr{C}$ is a Lagrangian submanifold of $T^{*} \mathbb{R}^{2}$ whose boundary consists of two components, namely

$$
\left\{\pi^{+}(x, \xi):(x, \xi) \in \Lambda(\omega)\right\}
$$

and the cosphere bundle

$$
S^{*} C(\omega)=\left\{(y, \eta) \in T^{*} \mathbb{R}^{2}: y \in C(\omega),|\eta|=1\right\} .
$$

By Stokes theorem,

$$
\ell(\omega)=\int_{S^{*} C(\omega)} \eta d y=\int_{\Lambda(\omega)} \xi d x=2 \pi \mathscr{J}(\omega),
$$

since the map $\chi=\psi^{0} \circ \psi$ conjugating $B$ to its symplectic normal form (3.11) and (3.12) is exact symplectic. This proves the first part of the claim.

To prove the second equality in (4.1) we use a symplectic trick which is due to Guillemin and Melrose [9] and Colin de Verdière [4]. Denote by $\sigma_{0}=I d \varphi$ the symplectic one-form in $T^{*} \mathbb{T}$. Since $B^{0}=\chi^{-1} \circ B \circ \chi$ is an exact symplectic map with a generating function $G^{0}(\varphi, I)=\tau(I)+Q^{0}(\varphi, I)$ given by Theorem 3.1, it is easy to see that

$$
\left(B^{0}\right)^{*} \sigma_{0}-\sigma_{0}=d f
$$


where

$$
f(\varphi, I)=I \tau^{\prime}(I)-\tau(I)+F(\varphi, I), \quad(\varphi, I) \in \mathbb{T} \times \Gamma,
$$

and the function

$$
F(\varphi, I)=I \frac{\partial Q^{0}}{\partial I}(\varphi, I)-Q^{0}(\varphi, I)
$$

has a zero of infinite order on the Cantor set $\mathbb{T} \times E$ in view of (3.12). On the other hand, (2.1) implies

$$
B^{*} \sigma-\sigma=d T^{0}, \quad \sigma=\xi d x,
$$

on $\Sigma$, where $T^{0}(x, \xi)=T(x, y(x, \xi))=|x-y(x, \xi)|$ and $y(x, \xi)$ is the first component of $B(x, \xi)$. Since $\chi$ is exact symplectic,

$$
\chi^{*} \sigma-\sigma^{0}=d \Phi, \quad \Phi \in C^{\infty}\left(T^{*} \mathbb{T}\right) .
$$

Now, (4.2), (4.4) and (4.5) yield together the following useful equality:

$$
f(\varphi, I)=T^{0}(\chi(\varphi, I))+\Phi(\varphi, I)-\Phi\left(B^{0}(\varphi, I)\right)+C, \quad(\varphi, I) \in \mathbb{A},
$$

where $C$ is a constant. Taking $I=l=\ell / 2 \pi$ we get $C=0$.

We are ready to prove the second equality in Proposition 4.1. Take $g^{0} \in \Lambda(\omega)$ and consider the orbit $g$ of $B$ defined by $g^{j}=B^{j} g^{0}=\left(x_{\jmath}, \xi_{\jmath}\right), j=0,1, \ldots$ Denote by

$$
\tilde{g}^{j}=\left(\varphi^{\jmath}, \mathscr{J}(\omega)\right)=\chi^{-1}\left(g^{j}\right), \quad j=0,1, \ldots,
$$

the corresponding orbit of $B^{0}$. For any $k \in N$ denote by $m_{k}$ the number of rotations that a point makes moving around $\partial \Omega$ in a positive direction from $x_{0}$ to $x_{k}$ and passing successively through each $x_{j}, j \leq k$. Then

$$
t(\omega)=\lim _{k \rightarrow \infty}\left(\frac{1}{k} \sum_{j=0}^{k} T^{0}\left(g^{j}\right)-\frac{m_{k}}{k} \ell(\omega)\right) .
$$

On the other hand, (3.12), (4.3) and (4.6) imply

$T^{0}\left(g^{j}\right)=2 \pi \omega \mathscr{J}(\omega)-\tau(\mathscr{J}(\omega))+\Phi(\varphi+2 \pi j \omega, \mathscr{J}(\omega))-\Phi(\varphi+2 \pi(j+1) \omega, \mathscr{J}(\omega))$.

Hence, the average action on $g=\left(g_{0}, g_{1}, \ldots\right)$ is equal to

$$
\lim _{k \rightarrow \infty}\left(\frac{1}{k} \sum_{j=0}^{k} T^{0}\left(g^{j}\right)\right)=2 \pi \omega \mathscr{J}(\omega)-\tau(\mathscr{J}(\omega)),
$$

the right-hand side being just the Legendre transform of $\tau(I) / 2 \pi$ times $2 \pi$. Moreover,

$$
\lim _{k \rightarrow \infty}\left(\frac{m_{k}}{k}\right)=\omega
$$

while $\ell(\omega)=2 \pi \mathscr{J}(\omega)$. Now, (4.7) and (4.8) yield together the second equality in (4.1). This completes the proof of the proposition.

Consider the approximated interpolating Hamiltonian $h(x, \xi)=K\left(\chi^{-1}(x, \xi)\right)$ of $B$ introduced by Corollary 3.2. For $r>0$ small enough we set as in Sect. 3

$$
M_{r}=\{(x, \xi) \in \Sigma: h(x, \xi)=r\}
$$


and denote

$$
\nu(r)=\int_{M_{r}} d t
$$

where the Poisson bracket

$$
\{h, t\}=H_{h} t=1 .
$$

It is easy to show that the set of Taylor coefficients of $\nu(r)$ at $r=0$ is algebraically equivalent to the set of Taylor coefficients of $K(I)$ at $I=l$. Indeed, performing a symplectic change of the variables $(x, \xi)=\chi(\varphi, I),(\varphi, I) \in \mathbb{T} \times \Gamma$, and using (4.10) we easily get

$$
K^{\prime}(I) \nu(K(I))=2 \pi, \quad I \in E .
$$

Denote by $\mathscr{B}$ the function inverse to $I \rightarrow K(I)$ and set $\widetilde{E}=\{K(I): I \in E\}$. Then $0 \in \widetilde{E}$ and (4.11) implies

$$
\nu(r)=2 \pi \mathscr{R}^{\prime}(r), \quad r \in \widetilde{E} .
$$

The Taylor coefficients of $\nu(t)$ at $t=0$, also called integral invariants, have been investigated by Marvizi and Melrose [16]. They are given by integrals on $\partial \Omega$ of certain polynomials of the curvature $\kappa(x)$ of $\partial \Omega$ and its derivatives. In particular, (4.6) in [16] and (4.12) yield together

$$
\begin{aligned}
& \mathscr{R}^{\prime}(0)=-\frac{1}{\pi} \int_{0}^{\ell} \kappa(x)^{2 / 3} d x, \\
& \mathscr{R}^{\prime \prime}(0)=\frac{1}{2160 \pi} \int_{0}^{\ell}\left(9 \kappa(x)^{4 / 3}+8 \kappa(x)^{-8 / 3} \kappa^{\prime}(x)^{2}\right) d x .
\end{aligned}
$$

\section{Marked Length Spectrum and Asymptotics of the Average Action}

Fix $b>0$ such that $\partial \Omega_{s}$ is strictly convex for any $0 \leq s \leq b$. Consider the set $\mathscr{G}\left(\Omega_{s}, m, n\right)$ of the periodic broken geodesics $\gamma$ of $\Omega_{s}$ with $n \geq 2$ vertices and winding number $m \leq n / 2$ and denote by $\mathscr{L}\left(\Omega_{s}, m, n\right)$ the set of lengths of all $\gamma$ in $\mathscr{G}\left(\Omega_{s}, m, n\right)$. The set $\mathscr{L}\left(\Omega_{s}, m, n\right)$ is compact and we define the marked length spectrum of $\Omega_{s}$ as a map that assigns to any two integers $(m, n), 1 \leq m \leq n / 2$, the maximal length

$$
T_{m n}(s)=\max \left\{t: t \in \mathscr{L}\left(\Omega_{s}, m, n\right)\right\}
$$

Following an idea due to $G$. Birkhoff we easily prove

Lemma 5.1. The set $\mathscr{C}\left(\Omega_{s}, m, n\right), 1 \leq m \leq n / 2$, is not empty for any $s \in[0, b]$. The function

$$
[0, b] \ni s \rightarrow T_{m n}(s)
$$

is continuous. 
Proof. As above consider $\Omega_{s}$ as $\Omega=\Omega_{0}$ equipped with a suitable Riemannian metric $\|\cdot\|_{s}^{2}$ which depends continuously on $s$. Denote by $\theta: \mathbb{R} \rightarrow \partial \Omega$ a smooth covering of $\partial \Omega, \theta(x+1)=\theta(x), x \in \mathbb{R}$. Consider the function

$$
\begin{aligned}
S_{s}\left(x_{1}, \ldots, x_{n}\right) & =\left\|\theta\left(x_{1}\right)-\theta\left(x_{2}\right)\right\|_{s}^{2}+\cdots+\left\|\theta\left(x_{n}\right)-\theta\left(x_{n+1}\right)\right\|_{s}^{2}, \\
x_{n+1} & =x_{1}+m,
\end{aligned}
$$

and set

$$
M=\left\{\left(x_{1}, \ldots, x_{n}\right) \in \mathbb{R}^{n}: x_{1} \leq x_{2} \leq \cdots \leq x_{n} \leq x_{n+1}=x_{1}+m, x_{j+1}-x_{j} \leq 1\right\} .
$$

Obviously $S_{s}$ is a continuous and periodic function in $M$ with a period $e=(1, \ldots, 1)$. Moreover, the factor space $M / \mathbb{Z} e$ is compact. The triangle inequality shows that the set

$$
M_{s}^{\max }=\left\{p \in M: S_{s}(p)=\max _{M} S_{s}\right\}
$$

consists only of points internal for $M$. Hence, $S_{s}$ is smooth on $M_{s}^{\max }$. Moreover, $\nabla S_{s}(p)=0$ at $p=\left(x_{1}, \ldots, x_{n}\right) \in M_{s}$ if and only if $\theta\left(x_{1}\right), \ldots, \theta\left(x_{n}\right)$ are successive vertices of a closed broken geodesic of $\Omega_{s}$ which belongs to $\mathscr{G}\left(\Omega_{s}, m, n\right)$. Therefore, the set $\mathscr{G}\left(\Omega_{s}, m, n\right)$ is not empty for any $s \in[a, b]$. Moreover,

$$
T_{m n}(s)=\sup \left\{S_{s}(p): p \in M\right\}
$$

is continuous in $s \in[0, b]$.

Lemma 5.2. The Lebesgue measure of $\mathscr{L}\left(\Omega_{s}\right)$ is zero.

Proof. Let $\gamma$ be a periodic broken geodesic in $\mathscr{G}\left(\Omega_{s}, m, n\right)$. Then length $(\gamma)=S_{s}(p)$ for some $p \in M$ such that $\nabla S_{s}(p)=0$. Applying Sard's theorem we obtain that $\mathscr{L}\left(\Omega_{s}, m, n\right)$ has Lebesgue measure zero which proves the claim.

Using (2.3), Lemma 5.1 and Lemma 5.2 we easily obtain

$$
T_{m n}(s)=T_{m n}(0), \quad s \in[0, b] .
$$

Indeed, according to (2.3) the continuous function $T_{m n}(s)$ takes values in $\mathscr{L}\left(\Omega_{0}\right)$ which does not contain intervals in view of Lemma 5.2. Hence, $T_{m n}(s)$ should be constant in $[0, b]$. We have proved that if the length spectrum remains constant along a continuous deformation of $\Omega_{s}$ then so does the marked length spectrum. We are going to show that the marked length spectrum determines uniquely the vector function (1.1).

First we evaluate the average action on the periodic orbits of the billiard ball map. To simplify the notations we drop the index $s$. Consider the set $\Gamma(m, n)$ of periodic orbits $g=\left(g_{1}, \ldots, g_{n}\right)$ of $B$ of period $n$ and winding number $m$. Any such orbit gives rise to a periodic broken geodesic in $\mathscr{G}(\Omega, m, n)$. Denote by $L(g)$ the length of the periodic broken geodesic of $\Omega$ associated with the periodic orbit $g$ of $B$.

According to (4.8), the average action of any orbit $\left\{g_{0}, g_{1}, \ldots\right\}, g_{j}=B^{j}\left(g_{0}\right)$, $g_{0} \in \Lambda(\omega)$, of $B$ on the invariant circle $\Lambda(\omega), \omega \in \Theta$, is given by the Legendre transform

$$
\mathscr{T}(\omega)=\omega \mathscr{J}(\omega)-\tau(\mathscr{J}(\omega)) / 2 \pi
$$

of $\tau(I) / 2 \pi$. Note that $\mathscr{T}(\omega)$ can be extended to a smooth odd function in $\mathbb{R}$ since 
$\mathscr{J}(\omega)$ is even, $\tau(I)=-\frac{4}{3} K(I)^{3 / 2}, K\left(l_{0}\right)=0, K^{\prime}\left(l_{0}\right)<0$ and (3.16) holds. Moreover,

$$
2 \pi \mathscr{T}(\omega)=\omega \ell(\omega)+t(\omega), \quad \forall \omega \in \Theta
$$

in view of Proposition 4.1.

We impose the following condition on the pair $(m, n) \in \mathbb{N}^{2}$ :

$$
\operatorname{dist}(m / n, \Theta) \leq n^{-1 / 2}, \quad 1 \leq m \leq n / 2,
$$

where $\operatorname{dist}(z, \Theta)$ is the distance between $z$ and $\Theta$. Here is the main result in this section:

Theorem 5.1. For any $(m, n) \in \mathbb{N}^{2}$ satisfying (5.4) and any $g \in \Gamma(m, n)$ we have

$$
|L(g) / n-2 \pi \mathscr{T}(m / n)| \leq C_{N} n^{-N}, \quad \forall N>0
$$

where $C_{N}$ is a positive constant which depends only on $N, B$, and on the symplectic transformation $\chi=\psi^{0} \circ \psi$.

Proof of Theorem 2.1. Fix $\omega \in \Theta$ and choose a sequence $\left(m_{j}, n_{\jmath}\right) \in \mathbb{N}^{2}, j \in \mathbb{N}$, satisfying (5.4). For any $j \in \mathbb{N}$ pick a periodic orbit $g_{s}^{j} \in \Gamma_{s}\left(m_{j}, n_{j}\right)$ of $B_{s}$ such that

$$
L_{s}\left(g_{s}^{\jmath}\right)=T_{m_{\jmath} n_{\jmath}}(s), \quad s \in[0, b] .
$$

Theorem 5.1 yields

$$
2 \pi \mathscr{T}_{s}(\omega)=\lim _{j \rightarrow \infty}\left(T_{m_{j} n_{j}}(s) / n_{\jmath}\right), \quad s \in[0, b],
$$

and taking into account (5.1) we obtain

$$
\mathscr{T}_{s}(\omega)=\mathscr{T}_{0}(\omega), \quad \forall \omega \in \Theta, \forall s \in[0, b]
$$

Since $\Theta$ has no isolated points, differentiating the last equality with respect to $\omega$ we obtain

$$
\mathscr{J}_{s}(\omega)=\mathscr{Z}_{0}(\omega), \quad K_{s}\left(\mathscr{Z}_{0}(\omega)\right)=K_{0}\left(\mathscr{Z}_{0}(\omega)\right), \quad \forall \omega \in \Theta, \quad \forall s \in[0, b],
$$

which proves (2.6). In particular,

$$
E_{s}=E_{0}, \quad K_{s}(I)=K_{0}(I), \quad \forall I \in E_{0},
$$

and using Theorem 3.1 we prove (ii), Theorem 2.1 for $s \in[0, b]$.

It remains to show that $\Omega_{s}$ is strictly convex for any $s \in[0,1]$. Suppose that $\Omega_{s}$ is strictly convex for $s<b_{0}$ but only convex for $s=b_{0}$. Consider the function $\mathscr{B}_{s}(r)$ inverse to $r=K_{s}(I)$ and set $\widetilde{E}_{s}=\left\{K_{s}(I): I \in E_{s}\right\}$. Then (5.6) yields

$$
\widetilde{E}_{s}=\widetilde{E}_{0}, \quad \mathscr{B}_{s}(r)=\mathscr{R}_{0}(r), \quad \forall r \in \widetilde{E}_{0}
$$

and since $0 \in \widetilde{E}_{0}$ we obtain

$$
\mathscr{R}_{s}^{\prime}(0)=\mathscr{R}_{0}^{\prime}(0), \quad \mathscr{R}_{s}^{\prime \prime}(0)=\mathscr{R}^{\prime \prime}(0), \quad s \in\left[0, b_{0}\right) .
$$

Denote by $\kappa_{s}(x), x \in \partial \Omega_{s}$, the curvature of $\partial \Omega_{s}$, set $f_{s}(x)=\kappa_{s}(x)^{-1 / 3}$ for $s<b_{0}$, and define $f_{b_{0}}(x)=\kappa_{b_{0}}(x)^{-1 / 3}$ if $\kappa_{b_{0}}(x) \neq 0, f_{b_{0}}(x)=+\infty$ if $\kappa_{b_{0}}(x)=0$. The second equality of (5.7) and (4.14) yield together

$$
\int_{\partial \Omega_{s}}\left|f_{s}^{\prime}(x)\right|^{2} d x \leq C, \quad s \in\left[0, b_{0}\right) .
$$


On the other hand, (4.13) and the first equality of (5.7) imply

$$
\kappa_{s}\left(x_{s}\right)>C_{1}>0, \quad s \in\left[0, b_{0}\right),
$$

for some $x_{s} \in \partial \Omega_{s}$. Then $0<f_{s}\left(x_{s}\right) \leq C_{2}, s \in\left[0, b_{0}\right)$, and using Taylor's formula and (5.8) we obtain the estimate

$$
\int_{\partial \Omega_{s}}\left(\left|f_{s}(x)\right|^{2}+\left|f_{s}^{\prime}(x)\right|^{2}\right) d x \leq C_{3}, \quad s \in\left[0, b_{0}\right),
$$

which means that $\left\{f_{s}: s \in\left[0, b_{0}\right)\right\}$ is a compact subset of $L^{2}(\partial \Omega)$ (we regard $\partial \Omega_{s}$ as $\partial \Omega$ equipped with a suitable Riemannian metric). In particular, $f_{b_{0}} \in L^{2}(\partial \Omega)$. On the other hand, the curvature $k_{b_{0}}(x) \geq 0$ and it has a zero of at least second order at some $x_{0} \in \partial \Omega$. Hence,

$$
\left|f_{b_{0}}(x)\right| \geq C\left|x-x_{0}\right|^{-2 / 3}
$$

in any local coordinates in a neighborhood of $x_{0}$ in $\partial \Omega$ which implies $f_{b_{0}} \notin L^{2}(\partial \Omega)$. Hence, $\partial \Omega_{s}$ is strictly convex for any $s \in[0,1]$. The proof of Theorem 2.1 is complete.

Proof of Theorem 5.1. The proof is based on a suitable approximation of $\left(B^{0}\right)^{j}(\varphi, I)$, $j \leq n$, where $B^{0}$ is introduced by Theorem 3.1. Fix $\varepsilon>0$ and consider a neighborhood

$$
V=\{\varrho \in \Sigma: 0 \leq h(\varrho) \leq \varepsilon\}
$$

of $\partial \Sigma_{s}^{+}$in $\Sigma$, where $h$ is the approximated interpolating Hamiltonian of $B$ introduced by Corollary 3.2. There exists $\varepsilon_{0}>0$ such that if $m / n<\varepsilon_{0}$ and $\left(g_{1}, \ldots, g_{n}\right) \in$ $\Gamma(m, n)$, then $g_{j} \in V$ for each $j \leq n$. Indeed, denote by $t$ the maximal length of the segments with end points $x_{j}$ and $x_{j+1}, j=1, \ldots, n$, where $g_{j}=\left(x_{j}, \xi_{j}\right)$, $x_{n+1}=x_{1}$. Then $t<m \ell_{0} / n<\varepsilon_{0} \ell_{0}$ which implies $g_{j} \in V$ if $\varepsilon_{0}$ is sufficiently small. Let $\omega \in \Theta \subset\left[0, \omega_{0}\right], \omega_{0}<\varepsilon_{0}$ and $m / n \leq \varepsilon_{0}$. We have $g_{j} \in V, j \leq n$, for any periodic orbit $g=\left(g_{1}, \ldots, g_{n}\right) \in \Gamma(m, n)$. Let

$$
\tilde{g}=\left(\widetilde{g}_{1}, \ldots, \tilde{g}_{n}\right), \quad \tilde{g}=\left(\varphi_{j}, I_{j}\right)=\chi^{-1}\left(g_{j}\right), \quad \chi=\psi^{0} \circ \psi
$$

Then $\widetilde{g}$ is a periodic orbit of $B^{0}$ of period $n$ and winding number $m$, and $\widetilde{g}_{j} \in \mathbb{T} \times \Gamma$, $\Gamma=\left(l_{0}-\varepsilon, l_{0}\right)$. According to Theorem 3.1, the map $B^{0}$ has the form

$$
B^{0}(\varphi, I)=\left(\varphi+\tau^{\prime}(I)+Q_{1}^{0}(\varphi, I), I+Q_{2}^{0}(\varphi, I)\right)
$$

where $Q_{1}^{0}$ and $Q_{2}^{0}$ have a zero of infinite order at $\mathbb{T} \times E$ and $E=\mathscr{J}(\Theta) \subset\left[l_{0}-\varepsilon, l_{0}\right]$. We denote by $V_{n}$ and $J_{n}$ suitable neighborhoods of $\Theta$ and $E$,

$$
V_{n}=\left\{\xi \in\left(\frac{1}{2 n}, \omega_{0}\right): \operatorname{dist}(\xi, \Theta) \leq 2 n^{-1 / 2}\right\}, \quad J_{n}=\mathscr{J}\left(V_{n}\right) .
$$

Taking into account (3.16) we find a positive constant $C_{0}>0$ such that

$$
\operatorname{dist}(I, E) \leq C_{0} n^{-1 / 2} \quad \text { and } \quad l_{0}-I \geq C_{0}^{-1} n^{-2}, \quad \forall I \in J_{n}, \quad \forall n \in \mathbb{Z}_{+} .
$$


Proposition 5.1. For any integers $N \geq 1, \alpha \geq 0$, and $0 \leq \beta \leq N$, there exists a constant $C_{N \alpha}$ such that

$$
\left|\partial_{\varphi}^{\alpha} \partial_{I}^{\beta}\left(\left(B^{0}\right)^{j}(\varphi, I)-\left(\varphi+j \tau^{\prime}(I), I\right)\right)\right| \leq C_{N \alpha} n^{-N}, \quad(\varphi, I) \in \mathbb{T} \times J_{n}
$$

for any $1 \leq j \leq n$ and any positive integer $n$.

Proof. Set $U(\varphi, \xi)=(\varphi, \mathscr{J}(\xi)),(\varphi, \xi) \in \mathbb{T} \times \mathbb{R}$, and consider the map $B=$ $U^{-1} \circ B^{0} \circ U$ in $\mathbb{T} \times\left(0, \omega_{0}\right)$. We have

$$
B(\varphi, \xi)=(\varphi+2 \pi \xi, \xi)+R(\varphi, \xi)
$$

where $R=\left(R_{1}, R_{2}\right)$ can be extended as a smooth mapping in $T \times \mathbb{R}$ across $\xi=0$. Indeed, we have

$$
\begin{aligned}
& R_{1}(\varphi, \xi)=Q_{1}^{0}(\varphi, \mathscr{J}(\xi)), \\
& R_{2}(\varphi, \xi)=\frac{1}{2 \pi} \tau^{\prime}\left(\mathscr{J}(\xi)+Q_{2}^{0}(\varphi, \mathscr{J}(\xi))-\xi,\right.
\end{aligned}
$$

where $Q_{j}^{0}, j=1,2$, are given by (5.9), they are smooth in $\mathbb{T} \times \mathbb{R}$, and have a zero of infinite order at $\mathbb{T} \times E$. On the other hand, the singularity of $\tau^{\prime}$ at $I=l$ is described by

$$
\tau^{\prime}(I)=-2 K(I)^{1 / 2} K^{\prime}(I), \quad K(l)=0, \quad K^{\prime}(l)<0
$$

and we prove easily that $R_{\jmath}$ are smooth at $\xi=0$ since $l \in E$. Moreover,

$$
\left|\partial_{\varphi}^{\alpha} \partial_{\xi}^{\beta} R_{j}(\varphi, \xi)\right| \leq C_{N \alpha \beta} n^{-3 N}, \quad j=1,2
$$

for any $(\varphi, \xi) \in \mathbb{T} \times \mathbb{R}$ such that $\operatorname{dist}(\Theta, \xi) \leq 3 n^{-1 / 2}$. Set

$$
B^{j}(\varphi, \xi)=\left(\Phi_{\jmath}(\varphi, \xi), \Xi_{\jmath}(\varphi, \xi)\right), \quad(\varphi, \xi) \in \mathbb{T} \times V_{n}, \quad j \leq n .
$$

First we prove by induction with respect to $j \leq n$ the inequalities

$$
\begin{aligned}
\left|\Xi_{j}-\xi\right| & \leq j n^{-4}, \quad\left|\Phi_{j}-\varphi-2 \pi j \xi\right| \leq \pi j^{2} n^{-4}, \\
\operatorname{dist}\left(\Xi_{\jmath}, \Theta\right) & \leq 2 n^{-1 / 2}+j n^{-4}<3 n^{-1 / 2}, \quad j \leq n,
\end{aligned}
$$

for any $(\varphi, \xi) \in \mathbb{T} \times V_{n}$ and $n \geq n_{1}$, where $n_{1}$ is sufficiently large. In the same way, making use of the third inequality in (5.13) as well as of (5.12) we obtain

$$
\left|\partial_{\varphi}^{\alpha} \partial_{\xi}^{\beta}\left(\Xi_{j}(\varphi, \xi)-\xi\right)\right|+\left|\partial_{\varphi}^{\alpha} \partial_{\xi}^{\beta}\left(\Phi_{j}(\varphi, \xi)-\varphi-2 \pi j \xi\right)\right| \leq C_{N \alpha \beta} n^{-3 N}, \quad 1 \leq j \leq n,
$$

in $(\varphi, \xi) \in \mathbb{T} \times V_{n}$ for any nonnegative integers $\alpha, \beta$, and $N$. Conjugating $B$ with $U$ and using the estimate

$$
\left|\partial_{I}^{\beta} \tau(I)\right| \leq C_{\beta} n^{2 \beta}, \quad I \in J_{n},
$$

which follows from (5.10) we complete the proof of Proposition 5.1.

Consider the set $W_{n}$ of all $\left(\varphi^{\prime}, \varphi\right) \in \mathbb{R}^{2}$ such that

$$
\left(\varphi^{\prime}-\varphi\right) / 2 \pi n \in V_{n}
$$

Set

$$
P^{0}(\varphi, I)=\left(B^{0}\right)^{n}(\varphi, I)=\left(\varphi+n \tau^{\prime}(I), I\right)+Q(\varphi, I)
$$


where $Q=\left(Q_{1}, Q_{2}\right)$ is a smooth function in $\mathbb{T} \times \mathbb{R}$. Using Proposition 5.1 we solve the equation

$$
\varphi^{\prime}=\varphi+n \tau^{\prime}(I)+Q_{1}(\varphi, I)
$$

with respect to $I \in J_{n}$ when $\left(\varphi^{\prime}, \varphi\right) \in W_{n}$ and $n$ is sufficiently large. This equation is equivalent to

$$
I=\mathscr{J}\left(\left(\varphi^{\prime}-\varphi\right) / 2 \pi n\right)+Q_{n}\left(\varphi^{\prime}, \varphi, I\right), \quad I \in J_{n}, \quad\left(\varphi^{\prime}, \varphi\right) \in W_{n},
$$

where, in view of (5.11) we have

$$
\left|\partial_{\varphi^{\prime}}^{\alpha} \partial_{\varphi}^{\beta} \partial_{I}^{p} Q_{n}\left(\varphi^{\prime}, \varphi, I\right)\right| \leq C_{N \alpha \beta} n^{-N} \quad \text { in } \quad W_{n} \times J_{n}
$$

for any $N$ and any indices $(\alpha, \beta, p) \in \mathbb{Z}_{+}^{3}, p \leq N$. Hence, (5.14) can be solved by successive iterates for $n \geq n_{0}$ and $n_{0}$ sufficiently large. Denote by $I\left(\varphi^{\prime}, \varphi\right)$, $\left(\varphi^{\prime}, \varphi\right) \in W_{n}, n \geq n_{0}$, the solution of (5.14). Then

$$
\left|I\left(\varphi^{\prime}, \varphi\right)-\mathscr{J}\left(\left(\varphi^{\prime}-\varphi\right) / 2 \pi n\right)\right| \leq C_{N} n^{-N}, \quad\left(\varphi^{\prime}, \varphi\right) \in W_{n}
$$

In particular, $\operatorname{graph}\left(P^{0}\right)$ can be parameterized over $\mathbb{T} \times J_{n}$ by $\left(\varphi^{\prime}, \varphi\right) \in W_{n}$ as follows:

$$
\operatorname{graph}\left(P^{0}\right)=\left\{\left(\varphi^{\prime}, \frac{\partial H_{n}}{\partial \varphi^{\prime}}\left(\varphi^{\prime}, \varphi\right), \varphi,-\frac{\partial H_{n}}{\partial \varphi}\left(\varphi^{\prime}, \varphi\right)\right):\left(\varphi^{\prime}, \varphi\right) \in W_{n}\right\}
$$

where $H_{n}$ is a smooth and $2 \pi$-periodic function on $\mathbb{R}^{2}$ satisfying the equality

$$
\frac{\partial H_{n}}{\partial \varphi}\left(\varphi^{\prime}, \varphi\right)=-I\left(\varphi^{\prime}, \varphi\right)
$$

Choose $m \in N$ and suppose that (5.4) holds for the pair $(m, n) \in \mathbb{N}^{2}, n \geq n_{0}$. Then $(\varphi+2 \pi m, \varphi) \in W_{n}$ for any $\varphi \in \mathbb{R}$. Set

$$
h(\varphi)=h_{m n}(\varphi)=H_{n}(\varphi+2 \pi m, \varphi), \quad \varphi \in \mathbb{R} .
$$

The function $h(\varphi)$ is smooth and $2 \pi$-periodic in $\mathbb{R}$ - According to (5.16) there is oneone correspondence between the critical points of $h^{m n}$ in $\mathbb{T}$ and the fixed points of $P^{0}$ in $\mathbb{T} \times \Gamma$ which is given by

$$
\operatorname{Crit}\left(h_{m n}\right) \ni \varphi \rightarrow g_{1}(\varphi)=(\varphi, I(\varphi+2 \pi m, \varphi)) \in \operatorname{Fix}\left(P^{0}\right) .
$$

Then

$$
\begin{gathered}
\operatorname{Crit}\left(h_{m n}\right) \ni \varphi \rightarrow g(\varphi)=\left(g_{1}(\varphi), \ldots, g_{n}(\varphi)\right) \in \Gamma(m, n), \\
g_{j}(\varphi)=B^{\jmath-1} g_{1}(\varphi), \quad j=1, \ldots, n,
\end{gathered}
$$

is one-one correspondence between the critical points of $h_{m n}$ and the periodic orbits of $B$ in $\Gamma(m, n)$, and (5.15) implies

$$
|I-\mathscr{J}(m / n)| \leq C_{N} n^{-N}
$$

at any periodic point $(\varphi, I)$ of $B^{0}$ of periodic $n$ and winding number $m$.

Take $g=\left(g_{1}, \ldots, g_{n}\right) \in \Gamma(m, n)$ and denote as before $\left(\varphi_{j}, I_{j}\right)=\chi^{-1}\left(g_{j}\right)$. Using (4.6) we obtain

$$
|L(g) / n-2 \pi \mathscr{T}(m / n)| \leq \frac{1}{n} \sum_{j=1}^{n}\left|f\left(\varphi_{j}, I_{j}\right)-2 \pi \mathscr{T}(m / n)\right| .
$$


On the other hand,

$$
\begin{aligned}
& \left|f\left(\varphi_{\jmath}, I_{\jmath}\right)-2 \pi \mathscr{T}(m / n)\right| \leq\left|\tau\left(I_{\jmath}\right)-\tau(\mathscr{J}(m / n))\right| \\
& \quad+\left|I_{j} \tau^{\prime}\left(I_{\jmath}\right)-\mathscr{J}(m / n) \tau^{\prime}(\mathscr{J}(m / n))\right|+C_{N} n^{-N} .
\end{aligned}
$$

We evaluate the right-hand side of the inequality above using Taylor's formula. We have $\left|\tau^{\prime}(I)\right| \leq C$ in $\Gamma$. Moreover,

$$
\tau^{\prime}\left(I_{j}\right)-\tau^{\prime}(\mathscr{J}(m / n))=\left(I_{j}-\mathscr{J}(m / n)\right) \tau^{\prime \prime}\left(\widetilde{I}_{j}\right)
$$

where

$$
\left|\tilde{I}_{\jmath}-\mathscr{J}(m / n)\right| \leq\left|I_{j}-\mathscr{J}(m / n)\right| \leq C_{N} n^{-N}
$$

according to (5.19). Then

$$
\left|\tau^{\prime \prime}\left(\tilde{I}_{j}\right)\right| \leq\left|K\left(\tilde{I}_{j}\right)^{-1 / 2} K^{\prime}\left(\widetilde{I}_{j}\right)^{2}\right|+C \leq C n
$$

Hence, $\left|f\left(\varphi_{j}, I_{j}\right)-2 \pi \mathscr{T}(m / n)\right| \leq C_{N} n^{-N}$ which completes the proof of Theorem 5.1.

The function $h$ introduced by (5.17) is going to play an important role in Sect. 6. Note that it is uniquely determined by (5.16) and (5.17) up to a constant and we normalize it by taking $H_{n}\left(\varphi_{0}, \varphi_{0}+2 \pi m\right)=L\left(g\left(\varphi_{0}\right)\right)$, where $\varphi_{0}$ is a point in $\operatorname{Crit}\left(h_{m n}\right)$. Then we obtain

Lemma 5.3. We have

$$
h_{m n}(\varphi)=L(g(\varphi)), \quad \forall \varphi \in \operatorname{Crit}\left(h_{m n}\right)
$$

Proof. Taking into account (4.4) we get

$$
\left(B^{n}\right)^{*}(\sigma)-\sigma=d T_{n}, \quad T_{n}=\sum_{\jmath=0}^{n}\left(B^{j}\right)^{*} T^{0} .
$$

On the other hand,

$$
\left(P^{0}\right)^{*}(I d \varphi)-I d \varphi=d H_{n}, \quad P^{0}=\left(\chi^{-1} \circ B \circ \chi\right)^{n},
$$

in $\mathbb{T} \times J_{n}$, and

$$
\chi^{*}(\sigma)-I d \varphi=d \Phi, \quad \Phi \in C^{\infty}(\mathbb{T} \times \mathbb{R}),
$$

since $\chi$ is exact symplectic. Set $T_{n}^{0}(\varphi, I)=T_{n}(\chi(\varphi, I))$. Taking into account the equalities above as well as the normalization of $H_{n}$ we easily obtain

$$
\begin{aligned}
H_{n}\left(\varphi^{\prime}, \varphi\right)= & T_{n}^{0}\left(\varphi, I\left(\varphi^{\prime}, \varphi\right)\right)-\Phi\left(P^{0}\left(\varphi, I\left(\varphi^{\prime}, \varphi\right)\right)\right) \\
& +\Phi\left(\varphi, I\left(\varphi^{\prime}, \varphi\right)\right), \quad\left(\varphi^{\prime}, \varphi\right) \in W_{n} .
\end{aligned}
$$

Using (5.17) and (5.18) we complete the proof of the lemma. 
The following result is a generalization of Theorem 5.15 in [16]

Corollary 5.1. Fix $m \in \mathbb{Z}_{+}$and pick arbitrarily $g_{m n} \in \Gamma(m, n)$. Then

$$
\left|L\left(g_{m n}\right)-\sum_{k=0}^{N} c_{m k} n^{-2 k}\right| \leq C_{N m} n^{-2 N-2},
$$

where

$$
c_{m k}=\frac{m^{2 k+1}}{(2 k+1) !} \mathscr{T}^{(2 k+1)}(0), \quad k=0,1, \ldots
$$

and $C_{N m}$ are positive constants.

Proof. Since $0 \in \Theta$, the pair $(m, n)$ satisfies (5.4) if $m$ is fixed and $n>n_{1}(m)$. Let us expand $\mathscr{T}(t)$ in Taylor series at $t=0$. The derivatives $\mathscr{T}^{(2 k)}(0)=0$ since the function $\mathscr{T}(t)$ is odd. Applying Theorem 5.1 we prove the assertion.

Remark 5.1. Since $0 \in \Theta$, we can write the coefficients $c_{m k}$ explicitly in terms of the Taylor series of $t=0$ of the restriction of the function $\mathscr{T}(\omega)$ on $\Theta$, which is given by (5.3). Moreover, the relation between $c_{m k}$ and the integral invariants of Marvizi and Melrose is explicitly given by (4.11) and (5.2).

Consider two strictly convex domains $\Omega_{0}$ and $\Omega_{1}$. Let $B_{\jmath}, j=0,1$ be the corresponding billiard ball maps. Choosing the constant $a_{0}$ in (3.8) sufficiently small we obtain for any $\omega \in \Theta$ an invariant curve $\Lambda_{j}(\omega)$ of $B_{j}, j=0,1$. The following statement is a discrete version of Theorem 2.1.

Theorem 5.2. Let $\Omega_{\jmath}, j=1,2$, be strictly convex domains in $\mathbb{R}^{2}$ and

$$
T_{m n}(0)=T_{m n}(1), \quad \forall(m, n), \quad 1 \leq m \leq n / 2 .
$$

Then

$$
\ell_{0}(\omega)=\ell_{1}(\omega), \quad t_{0}(\omega)=t_{1}(\omega), \quad \forall \omega \in \Theta
$$

and there exists an exact symplectic mapping $\chi: \Sigma_{0} \rightarrow \Sigma_{1}$ such that

$$
\chi\left(\Lambda_{0}(\omega)\right)=\Lambda_{1}(\omega) \text { and } \chi \circ B_{0}=B_{1} \circ \chi \text { on } \Lambda_{0}(\omega), \quad \forall \omega \in \Theta .
$$

Theorem 5.2 follows from Theorem 5.1 and the arguments at the end of the proof of Theorem 2.1.

\section{Spectral Invariants}

Let $\Omega$ be a bounded domain in $R^{2}$ with a smooth boundary $\partial \Omega$. Consider the Laplacian $\Delta$ in $\Omega$ with Dirichlet (Neumann) boundary conditions, and the related distribution $Z(t)$ defined by $(1.8)$.

Denote by $\mathscr{G}(\Omega)$ the set of all periodic generalized geodesics of $\Omega$ and consider the set $\mathscr{G}(\Omega ; m, n)$ of the periodic broken geodesics in $\Omega$ corresponding to the periodic orbits of $B$ in $\Gamma(m, n)$. Let $\mathscr{L}_{m n}(\Omega)$ be the set of lengths of all periodic broken geodesics in $\mathscr{G}(\Omega) \backslash \mathscr{G}(\Omega, m, n)$. In order to prevent cancellation of the singularity of $Z(t)$ created by the geodesics of maximal length $T_{m n}$ in $\mathscr{G}(\Omega ; m, n)$, we impose the following condition:

$$
T_{m n} \notin \mathscr{L}_{m n}(\Omega) \text {. }
$$

This condition is satisfied for generic domains $\Omega$ (see [18]). 
Theorem 6.1. Let $\Omega$ be a strictly convex bounded domain in $\mathbb{R}^{2}$ with a smooth boundary. Suppose that $(m, n) \in \mathbb{N}^{2}$ satisfies (5.4) and that (6.1) is fulfilled. Then

$$
T_{m n} \in \text { sing. supp. }(Z)
$$

holds if $n \geq n_{0}$ and $n_{0}=n_{0}(\Omega)$ is sufficiently large.

This statement generalizes Theorem 6.4 in [16] where it has been proved for $m=1$ and $n$ sufficiently large. The main idea in [16] is to find suitable representation of $Z(t)$ in a neighborhood of $T_{m n}$ as a Fourier integral on $\partial \Omega$ (see Proposition 6.11 in [16]) and then to apply a result of Soga [22]. When $m$ is fixed and $n \geq n_{1}(m)$ is sufficiently large Proposition 6.11 in [16] still holds. In the general case when $(m, n)$ satisfies (5.4) and $n \geq n_{0}(\Omega)$, we use another representation of $Z(t)$ in a neighborhood of $T_{m n}$ which is close to that obtained in Proposition 5.4, [21]. Consider the function $h(\varphi)=h_{m n}(\varphi), \varphi \in \mathbb{T}$, defined by (5.17). For any $z \in \mathbb{C}$ denote by $\operatorname{Re} z$ its real part.

Proposition 6.1. Suppose that ( $m, n$ ) satisfies (5.4), $n \geq n_{0}$, and that (6.1.) holds. If $n_{0}=n_{0}(\Omega)$ is sufficiently large, we have

$$
Z(t)=\int_{0}^{\infty} \int_{\mathbb{T}} \operatorname{Re}\left(\exp \left(i \tau(t-h(\varphi))+i \pi \mu_{n}\right) a(\varphi, \tau)\right) d \varphi d \tau+\widetilde{Z}(t),
$$

where $\widetilde{Z}(t)$ is smooth at $t=T_{m n}, \mu_{n}$ is a Maslov index, and $a(\varphi, \tau)$ is a classical symbol of order one with respect to $\tau$. Moreover, $a(\varphi, \tau)=0$ for $\tau \leq 1$ and the principal part of a is equal to $a_{1}(\varphi) \tau$ for $\tau \geq 2$, where $a_{1}>0$ on $\mathbb{T}$.

Applying Lemma 5.5 in [21] to the oscillatory integral given by Proposition 6.1 we prove Theorem 6.1.

Proof of Proposition 6.1. Consider the fundamental solution $E(t, x, y)$ of the mixed problem

$$
\begin{aligned}
\left(D_{t}^{2}-D_{x}^{2}\right) E & =0, \\
E_{\mid x \in \partial \Omega} & =0, \\
E(0, x, y) & =\delta(x-y), \\
\left(D_{t} E\right)(0, x, y) & =0,
\end{aligned}
$$

where $D_{t}=-i \partial / \partial t, D_{x}^{2}=D_{x_{1}}^{2}+D_{x_{2}}^{2}, D_{x_{\jmath}}=-i \partial / \partial x_{\jmath}, j=1,2$. The distribution $E$ is just the kernel of the operator $\cos (t \sqrt{\Delta})$. Denote by $E^{ \pm}$the Schwartz kernel of the operator $\exp (\mp i t \sqrt{\Delta})$ and consider

$$
Z^{ \pm}(t)=\int_{\Omega} E^{ \pm}(t, x, x) d x
$$

Then, $Z(t)=Z^{+}(t)+Z^{-}(t)$ and $Z^{-}=\overline{Z^{+}}$in a distribution sense where $\bar{z}$ is the complex conjugated number to $z \in \mathbb{C}$. As in (6.14), [16], using (6.1) we obtain

$$
Z^{+}(t)=\int_{\partial \Omega} K^{+}(t, x, x) d x+Z_{1}^{+}(t)
$$


where $Z_{1}^{+}$is smooth at $T_{m n}$ and $K^{+}$belongs to the Hörmander class

$$
I^{-1 / 4}\left(\mathbb{R} \times \partial \Omega \times \partial \Omega ; C_{+}^{\prime}\right)
$$

of Lagrangian distributions associated with the Lagrangian manifold

$$
\begin{aligned}
C_{+}^{\prime}= & \left\{(t, x, y ; \tau, \xi, \eta) \in T^{*}(\mathbb{R} \times \partial \Omega \times \partial \Omega): t=T_{n}(y,-\eta / \tau),\right. \\
& \left.(x, \xi / \tau)=B^{n}(y,-\eta / \tau), \tau>0\right\},
\end{aligned}
$$

where $T_{n}(y, \eta)$ is introduced in Lemma 5.3. Choose neighborhoods $V_{1} \subset V_{2} \subset C_{+}^{\prime}$ of the set

$$
C_{m n}=\left\{(t, x, y ; \tau, \xi, \eta) \in C_{+}^{\prime} ;(y,-\eta / \tau) \in \Gamma(m, n)\right\} .
$$

Without loss of generality we can suppose that the complete symbol of $K_{+}$vanishes outside $V_{2}$ while its principal symbol is a positive function in $V_{1}$ modulo a Maslov factor. Denote by $C_{0}^{\prime}$ a Lagrangian submanifold of $T^{*}(\partial \Omega \times \partial \Omega)$ associated with the graph of $B^{n}$,

$$
C_{0}^{\prime}=\left\{(x, y ; \xi, \eta) \in T^{*}(\partial \Omega \times \partial \Omega) ;(x, \xi)=B^{n}(y,-\eta),(y, \eta) \in \Sigma\right\},
$$

and let $V_{1}^{0} \subset V_{2}^{0} \subset C_{0}^{\prime}$ be neighborhoods of the set

$$
\left\{(x, y ; \xi, \eta) \in C_{0}^{\prime} ;(y,-\eta) \in \Gamma(m, n)\right\}
$$

As in Lemma 5.7, [21], we find a Fourier oscillatory integral $R(x, y, \tau)$ of the class

$$
I^{0}\left(\partial \Omega \times \partial \Omega ; C_{0}^{\prime}, \tau\right)
$$

such that

$$
K_{+}(t, x, y)=\int e^{-\imath t r} R(x, y, \tau) d \tau .
$$

Since $B^{n}$ is an exact symplectic mapping, the Liouville class of $C_{0}^{\prime}$ in $H^{1}\left(C_{0}^{\prime} ; \mathbb{R}\right)$ given by the restriction of the canonical symplectic one form of $T^{*}(\partial \Omega \times \partial \Omega)$ on $C_{0}^{\prime}$ is trivial. In this case there is a complete analogy between the theory of the Lagrangian distributions and the Fourier oscillatory integrals (see [7]). The only difference is that the principal symbol of a Fourier oscillatory integral associated with $C_{0}^{\prime}$ has an additional Liouville factor

$$
\exp (i \tau f(\varrho)), \quad \varrho \in C_{0}^{\prime}
$$

where $f$ is given by the restrictions on $C_{0}^{\prime}$ of suitable phase functions generating $C_{0}^{\prime}$. In our case, the principal symbol of $R$ is equal to

$$
\exp \left(i \tau T_{n}(y,-\eta)+i \pi \mu_{n}\right)
$$

times a positive function in $V_{1}^{0}$, the complete symbol of $R$ vanishes outside $V_{2}^{0}$ and $R(x, y, \tau)=O_{N}\left(\tau^{-N}\right)$ as $\tau \rightarrow-\infty$ for any $N>0$.

Denote by $R(\tau)$ the Fourier integral operator with a large parameter $\tau$ whose Schwartz kernel is $R(x, y, \tau)$. Using Theorem 3.1 (here $s$ is fixed) we shall conjugate $R(\tau)$ to a Fourier integral operator on $\mathbb{T}$ with a large parameter $\tau$ and phase function $h_{m n}(\varphi)$. Denote by $C_{1}^{\prime}$ the Lagrangian manifold

$$
C_{1}^{\prime}=\left\{(x, \varphi ; \xi, I) \in T^{*}(\partial \Omega \times \mathbb{T}) ;(x, \xi)=\chi(\varphi,-I)\right\}
$$

and observe that the Maslov bundle of $C_{1}^{\prime}$ is trivial since the projection

$$
C_{1}^{\prime} \ni(x, \varphi ; \xi, I) \rightarrow(x, \varphi) \in \partial \Omega \times \mathbb{T}
$$


is a diffeomorphism. Indeed, we have $\chi^{-1}(x, \xi)=(\Phi(x, \xi), \Xi(x, \xi))$, where $\partial \Xi / \partial \xi \neq 0$ on $\partial \Sigma_{+}$since the derivative with respect to $\xi$ of the approximated interpolating Hamiltonian $h(x, \xi)=K(\Xi(x, \xi))$ is different from zero on $\partial \Sigma_{+}$. We can replace $\chi$ by $\chi \circ \kappa$, where $\kappa(\varphi, I)=(\varphi+C I, I), C>0$. Then

$$
(\chi \circ \kappa)^{-1}(x, \xi)=(\Phi(x, \xi)-C \Xi(x, \xi), \Xi(x, \xi)),
$$

and we can solve the equation $\varphi=\Phi(x, \xi)-C \Xi(x, \xi)$ with respect to $\xi$ if $C$ is sufficiently big.

As in [21], Lemma 5.8, (see also [5]), we easily obtain

Lemma 6.1. Let $\Psi(\tau)$ be a pseudodifferential operator with a large parameter $\tau$ with symbol equal to one in $\mathbb{T} \times I_{n}$ and equal to zero outside a neighborhood of this set in $\mathbb{T} \times \Gamma$. There exists a Fourier integral operator $A(\tau)$ of the class $I^{0}\left(\partial \Omega \times \mathbb{T}, C_{1}^{\prime}, \tau\right)$ such that

$$
A^{*}(\tau) A(\tau)=\Psi(\tau)
$$

the principal symbol of $A(\tau)$ is equal to one on the lifting of $\mathbb{T} \times V_{n}$ in $C_{1}^{\prime}$ and the complete symbol of $A(\tau)$ vanishes outside a small neighborhood of it.

The operator

$$
R_{1}(\tau)=A^{*}(\tau) R(\tau) A(\tau): L^{2}(\mathbb{T}) \rightarrow L^{2}(\mathbb{T})
$$

has a distribution kernel $R_{1}\left(\varphi^{\prime}, \varphi, \tau\right)$ in $I^{0}\left(\mathbb{T} \times \mathbb{T}, C_{2}^{\prime}, \tau\right)$, where

$$
C_{2}^{\prime}=\left\{\left(\varphi^{\prime}, \varphi ; I^{\prime}, I\right) \in T^{*} \mathbb{T}^{2} ;\left(\varphi^{\prime}, I^{\prime}\right)=P^{0}(\varphi,-I)\right\}, \quad P^{0}=\left(B^{0}\right)^{n} .
$$

According to (5.16), $C_{2}^{\prime}$ is generated by $H_{n}\left(\varphi^{\prime}, \varphi\right)+C,\left(\varphi^{\prime}, \varphi\right) \in W_{n}$, where $C$ is constant. Hence we get

$$
R_{1}\left(\varphi^{\prime}, \varphi, \tau\right)=\exp \left(i \tau\left(H_{n}\left(\varphi^{\prime}, \varphi\right)+C\right)+i \pi \mu_{n}\right) b\left(\varphi^{\prime}, \varphi, \tau\right)
$$

where $b=0$ for $\tau<1$ and $b\left(\varphi^{\prime}, \varphi, \tau\right)=b_{1}\left(\varphi^{\prime}, \varphi\right) \tau+b_{0}\left(\varphi^{\prime}, \varphi\right)+\cdots$ is a classical symbol of order one as $\tau \rightarrow+\infty, b=0$ outside a neighborhood of $W_{n}$, and $b_{0}$ is a positive function in $W_{n}$. On the other hand, comparing the Liouville factors of $R(x, y, \tau)$ and $R_{1}\left(\varphi^{\prime}, \varphi, \tau\right)$, we obtain as in Sect. 5.4 in [21] and Sect. 3.2 in [3] that $C=0$.

The $L^{2}$-trace of $R(\tau)$ is equal to

$$
\operatorname{trace} R(\tau)=\operatorname{trace} R_{1}(\tau)=\int_{\mathbb{T}} \exp \left(i \tau h(\varphi)+i \pi \mu_{n}\right) b(\varphi+2 \pi m, \varphi, \tau) d \varphi,
$$

which completes the proof of Proposition 6.1.

Using Theorem 6.1 and certain arguments from Sect. 5 we prove that the invariant circles $\Lambda_{s}(\omega)$ and the restriction of the billiard ball map on them are spectral invariants of the Laplacian for suitable continuous deformations of a strictly convex domain. Define $\mathscr{T}_{m n}(s)$ as $T_{m n}(s)$ when $\Gamma_{s}(m, n)$ is not empty and set $\mathscr{T}_{m n}(s)=0$ otherwise. We say that the deformation (2.2) satisfies the condition ( $\mathscr{R})$, if for any pair of integers $(m, n), 1 \leq m \leq n / 2$, satisfying

$$
\operatorname{dist}(m / n, \Theta) \leq n^{-1 / 2}
$$

the relation

$$
\mathscr{T}_{m n}(s) \notin \mathscr{L}_{m n}\left(\Omega_{s}\right)
$$


is fulfilled for $s$ in a dense subset of $[0,1]$. Note that by definition $\mathscr{T}_{m n}(s)$ does not belong to the length spectrum of $\Omega_{s}$ if the set $\Gamma_{s}(m, n)$ is empty. Moreover, using arguments from [18] it could be proved that $(\mathscr{R})$ is generic for continuous deformations of the domain.

Theorem 6.2. Let $\Omega_{0}$ be a strictly convex bounded domain with a smooth boundary. Suppose that (2.2) is a continuous deformation of $\Omega_{0}$ satisfying (R) and that

$$
\operatorname{Spec}\left(\Delta_{s}\right)=\operatorname{Spec}\left(\Delta_{0}\right), \quad 0 \leq s \leq 1
$$

Then:

(i) $\Omega_{s}$ is strictly convex for any $s \in[0,1]$,

(ii) there exists a continuous family of smooth exact symplectic mappings

$$
\chi_{s}: T^{*} \partial \Omega_{0} \rightarrow T^{*} \partial \Omega_{s}
$$

such that

$$
\chi_{s}\left(\Lambda_{0}(\Omega)\right)=\Lambda_{s}(\omega), \text { and } \chi_{s} \circ B_{0}=B_{s} \circ \chi_{s} \text { on } \Lambda_{0}(\omega)
$$

for any $\omega \in \Theta_{0}$ and any $s \in[0,1]$,

(iii) for any $\omega \in \Theta_{0}$ there exists a continuous family of caustics $[0,1] \rightarrow C_{s}(\omega)$ in $\Omega_{s}$ and

$$
\ell_{s}(\omega)=\ell_{0}(\omega), \quad t_{s}(\omega)=t_{s}(\omega), \quad s \in[0,1] .
$$

Proof. Take $b>0$ such that $\Omega_{s}$ are strictly convex for any $s \in[0, b]$. Then $\mathscr{T}_{m n}(s)=T_{m n}(s)$ is continuous in $[0, b]$. Fix $\omega \in \Theta$ and suppose that the pair $(m, n) \in \mathbb{N}$ satisfies (5.4). We are going to prove that

$$
T_{m n}(s) \in \operatorname{sing} \text {. supp. } Z_{0}, \quad \forall s \in[0, b] .
$$

Take $s_{0} \in[0, b]$ and choose a sequence $s_{j}$ tending to $s_{0}$ such that $T_{m n}\left(s_{j}\right) \notin$ $\mathscr{L}_{m n}\left(\Omega_{s_{\jmath}}\right)$. Theorem 6.1 implies

$$
T_{m n}\left(s_{j}\right) \in \text { sing. supp. } Z_{s_{\jmath}} \text {. }
$$

On the other hand, $Z_{s}(t) \equiv Z_{0}(t)$ for any $s$ in view of (6.3) and we get (6.4) since sing. supp. $Z_{0}$ is a closed set and $T_{m n}(s)$ is continuous. Using the Poisson relation (1.9) we obtain

$$
T_{m n}(s) \in \mathscr{L}\left(\Omega_{0}\right), \quad \forall s \in[0, b]
$$

Hence,

$$
T_{m n}(s)=T_{m n}(0), \quad \forall s \in[0, b],
$$

and as in Theorem 2.1 we complete the proof of Theorem 6.2.

\section{Appendix}

We are going to prove Theorem 3.1. As in Sect. 3 we fix $N \in \mathbb{Z}_{+}$and $\mu>0$, and denote

$$
\varepsilon(\mu, N)=\varepsilon_{N} \mu^{M}, \quad M=N+2,
$$

where the positive constants $\varepsilon_{N}$ will be specified later. Fix $0<C_{0}<C<1, C_{1} \gg 1$ sufficiently large, set $\Gamma_{a}=\left[l_{0}-C_{1} a^{2}, l_{0}-C_{1}^{-1} a^{2}\right]$, and denote

$$
\Theta^{0}(a, \mu, N)=\Theta(a, \mu, N) \cap\left(C_{0} a, C_{0}^{-1} a\right), \quad 0<a<a_{0},
$$

where $C$ is fixed in (3.8). The following result is a counterpart of Theorem 1.1, [21]: 
Theorem A.1. Let $[0, b] \ni s \rightarrow B_{s} \in C^{\infty}(\mathbb{A}, \mathbb{A})$ be a continuous deformation of exact symplectic mappings satisfying the assumptions of Theorem 3.1 for any $s$ in $[0, b]$. Then for any $N \in \mathbb{Z}_{+}$there is $\varepsilon_{N}>0$, and for any $a \in(0, \varepsilon(\mu, N))$ there exist continuous in $s \in[0, b]$ families of exact symplectic mappings $\chi_{\text {sa }} \in C^{\infty}(\mathbb{T} \times \mathbb{R}, \mathbb{T} \times \mathbb{R})$ and functions $K_{s a} \in C^{\infty}(\mathbb{R}), Q_{s a} \in C^{\infty}(\mathbb{T} \times \mathbb{R})$ such that $K_{s a}(I)>0$ in $\Gamma$ and:

(i) the exact symplectic mapping $B_{s a}^{0}=\chi_{s a}^{-1} \circ B_{s} \circ \chi_{s a}$ is generated in $\mathbb{A}$ by

$$
\begin{aligned}
G_{s a}^{0}(\varphi, I) & =\tau_{s a}(I)+Q_{s a}^{0}(\varphi, I), \\
\tau_{s a}(I) & =-\frac{4}{3} K_{s a}(I)^{3 / 2}, \quad(\varphi, I) \in \mathbb{A},
\end{aligned}
$$

and

$$
Q_{s a}^{0}(\varphi, I)=0 \quad \text { on } \quad \mathbb{T} \times E_{s a},
$$

where $E_{s a}=\left\{I \in \Gamma: \tau_{s a}^{\prime}(I) / 2 \pi \in \Theta^{0}(a, \mu, N)\right\} \subset \Gamma_{a}$,

(ii) $\chi_{s a}=0$ outside $\mathbb{T} \times \Gamma_{a}$, and $K_{s a}, Q_{s a}^{0}$, and the generating function $S_{s a}(\theta, I)$ of $\chi_{\text {sa }}$ satisfy the estimates

$$
\begin{aligned}
\left|D_{I}^{\alpha}\left(K_{s a}(I)-\zeta_{s}(I)\right)\right|+\left|D_{I}^{\alpha} D_{\varphi}^{\beta} Q_{s a}^{0}(\varphi, I)\right| & \leq C_{\beta N}\left|l_{0}-I\right|^{N-\alpha} \\
\left|D_{I}^{\alpha} D_{\theta}^{\beta} S_{s a}^{0}(\theta, I)\right| & \leq C_{\beta N}\left|l_{0}-I\right|^{N-\alpha}
\end{aligned}
$$

in $\mathbb{T} \times\left[l_{0}-\varepsilon_{0}, l_{0}+\varepsilon_{0}\right], \varepsilon_{0}>0$, for $s \in[0, b]$ and any indices $0 \leq \alpha \leq N, \beta \geq 0$, where $C_{\beta N}$ depend neither on $s$ nor on $a$ and $\mu$.

The proof of Theorem A.1 is close to that of Theorem 1.1 in [21] and we are going only to sketch it. It is based on Theorem A, [19] and on an idea of R. Douady [6] (see also Appendix, [21]) to transform the initial problem for symplectic mappings to a similar problem for Hamiltonian systems.

First we write the generating function $G_{s}(\theta, r)$ of $B_{s}$ in the form

$$
G_{s}(\theta, r)=-\frac{4}{3} \zeta_{s}^{0}(r)^{3 / 2}+R_{s}(\theta, r), \quad(\theta, r) \in \mathbb{A}
$$

where $\zeta_{s}^{0}(r)$ is the Taylor polynomial of $\zeta_{s}(r)$ at $r=l_{0}$ up to order $M_{1}=N^{2}+4 N+5$ while $R_{s}$ satisfies

$$
\left|\partial_{\theta}^{\alpha} \partial_{r}^{\beta} R_{s}(\theta, r)\right| \leq C\left|r-l_{0}\right|^{M_{1}-\beta}, \quad(\theta, r) \in \mathbb{A},
$$

for any indices $\alpha \geq 0, \beta \geq 0$, and $s \in[0, b]$, and $R_{s}$ depends continuously on $s$ in $C^{\infty}(\mathbb{A})$. As in Sect. 3, we suppose that $R_{s}(\theta, r)=0$ for $r \in\left[l_{0}-\varepsilon, l_{0}-2 \varepsilon / 3\right]$. Fix $d=a^{2}$ and set

$$
\begin{aligned}
\mathbb{D} & =\Gamma \times\left(-\varepsilon_{0}, \varepsilon_{0}\right), \\
\mathbb{D}_{d} & =\left(l_{0}-C_{2} d, l_{0}-C_{2}^{-1} d\right) \times\left(-C_{2} d, C_{2} d\right),
\end{aligned}
$$

where $C_{2}>C_{1}>1$. Define $\widetilde{\mathbb{D}}_{d}$ the same way as $\mathbb{D}_{d}$ with a constant $C_{3}>C_{2}$ and set

$$
\widetilde{\mathbb{A}}=\mathbb{T}^{2} \times \mathbb{D}, \quad \widetilde{\mathbb{A}}_{d}=\mathbb{T}^{2} \times \widetilde{\mathbb{D}}_{d}
$$

Denote $y=\left(y_{1}, y_{2}\right) \in \mathbb{T}^{2}, \eta=\left(\eta_{1}, \eta_{2}\right) \in \mathbb{D}, y_{1}=\theta, \eta_{1}=r$ and $\tau_{s}^{0}(\eta)=-\frac{4}{3} \zeta_{s}^{0}\left(\eta_{1}\right)^{3 / 2}$. As in $[6,21]$, we first construct a Hamiltonian $\widetilde{H}_{s}(y, \eta)$ in $\widetilde{\mathbb{A}}$ close to $\widetilde{H}_{s}^{0}(\eta)=$ 
$2 \pi \eta_{2}+\tau_{s}^{0}\left(\eta_{1}\right)$ and such that the Poincare map corresponding to the Hamiltonian flow $F_{s}^{t}(\varrho)$ of $\widetilde{H}_{s}$ on the level surface $\left\{\widetilde{H}_{s}=0\right\}$ coincides with $B_{s}$. Set

$$
\mathbb{A}^{\prime}=\left\{(y, \eta) \in \widetilde{\mathbb{A}}: \widetilde{H}_{s}(\eta)=0, y_{2}=0\right\}
$$

and denote by $l_{s}: \mathbb{A} \rightarrow \mathbb{A}^{\prime}$ the inclusion map

$$
\iota_{s}\left(y_{1}, \eta_{1}\right)=\left(y_{1}, 0, \eta_{1},-\tau_{s}^{0}\left(\eta_{1}\right) / 2 \pi\right) .
$$

Taking into account (A.5) and (A.6) we prove as in [13] and [21]

Lemma A.1. There exists a continuous in $[0, b]$ family of Hamiltonians $\widetilde{H}_{s} \in C^{\infty}(\widetilde{\mathbb{A}})$ such that

$$
\left|D_{y}^{\alpha} D_{\eta}^{\beta}\left(\widetilde{H}_{s}(y, \eta)-\widetilde{H}_{s}^{0}(\eta)\right)\right| \leq C_{\alpha \beta}\left|\eta_{1}\right|^{M_{1}-|\beta|}
$$

$\widetilde{H}_{s}(y, \eta)=H_{s}^{0}(\eta)$ in a neighborhood of $\mathbb{A}^{\prime}$ as well as outside $\widetilde{\mathbb{A}}_{d}$,

$$
B_{s}=\iota_{s}^{-1} \circ F_{s}^{1} \circ \iota_{s} \text {. }
$$

Set $H_{s}^{0}=\widetilde{H}_{s}^{0}+\left(\widetilde{H}_{s}^{0}\right)^{2}, H_{s}^{\prime}=\widetilde{H}_{s}+\left(\widetilde{H}_{s}\right)^{2}$. Next we apply a KAM theorem to the pair $H_{s}^{0}, H_{s}^{\prime}$, which is a variant of Theorem A, [19], proved by J. Pöschel (see also Theorem 5.4 in [13]). As in [19] denote by $\sigma_{\gamma}(y, \eta)$ the map $(y, \eta) \rightarrow(y, \gamma \eta)$ and by $\|\cdot\|_{p, \widetilde{A}_{d}}$ the respective Hölder norms of the functions in $\widetilde{A}_{d}$ as well as

$$
\|f\|_{p, \widetilde{A}_{d} ; \gamma}=\left\|f \circ \sigma_{\gamma}\right\|_{p, \sigma_{\gamma}^{-1}\left(\tilde{A}_{d}\right)} .
$$

Denote by $\widetilde{\Gamma}$ the intersection of a neighborhood of $\left(0, l_{0}\right)$ in $\mathbb{C}^{2}$ with the half-plane $\left\{\operatorname{Re} z_{2}<l_{0}\right\}$ and set

$$
\mathbb{D}_{d}+\varrho=\left\{z \in \widetilde{\Gamma}:|z-\eta| \leq \varrho \text { for some } \eta \in \widetilde{\mathbb{D}}_{d}\right\}
$$

Next fix $\sigma>1, \mu>0$, as in Sect. 3, set $\gamma_{d}=\mu d^{N / 2}=\mu a^{N}$, and consider the Cantor set

$$
\Theta^{d}=\left\{\omega \in \mathbb{R}^{2}:|\langle\omega, k\rangle| \geq \gamma_{d}|k|^{-\sigma} \quad \text { for any } \quad k=\left(k_{1}, k_{2}\right) \in \mathbb{Z}^{2} \backslash\{0\}\right\} .
$$

Fix $s_{0} \in[0, b]$. The following KAM theorem is a variant of Theorem A in [19].

Theorem A.2. Let $[0, b] \ni s \rightarrow H_{s}^{0}(\eta)$ be a continuous family of analytic functions in $\widetilde{\Gamma}$ such that

$$
\left\|\partial H_{s}^{0} / \partial \eta^{2}\right\|_{\mathbb{D}_{d+\varrho}}, \quad\left\|\left(\partial H_{s}^{0} / \partial \eta^{2}\right)^{-1}\right\|_{\mathbb{D}_{d+\varrho}} \leq C d^{-1 / 2}, \quad d \in\left(0, d_{0}\right)
$$

where $\varrho=c d, 0<c<1, C>0$, and assume the map $\partial H_{s}^{\prime} / \partial \eta: \widetilde{\Gamma} \rightarrow \mathbb{C}^{2}$ to be invertible.

For any fixed $\lambda>\sigma+1>2$, and $\alpha>1, \alpha \notin \Lambda=\{i / \lambda+j: i, j \geq 0$ integer $\}$, there is a positive $\varepsilon$ independent of $d, \mu$ and s such that if $H_{s}^{\prime} \in C^{\infty}(\widetilde{\mathbb{A}})$ is continuous in $[0, b]$, and

$$
\left\|H_{s}^{\prime}-H_{s}^{0}\right\|_{p, \widetilde{\mathbb{A}}_{d} ; \gamma_{d}} \leq \gamma_{d}^{2} d^{2} \varepsilon, \quad p=\alpha \lambda+\lambda+\sigma,
$$


then:

(i) for any $d \in\left(0, d_{0}\right)$ there is a neighborhood $U\left(s_{0}\right)$ of $s_{0}$ and continuous with respect to $s \in U\left(s_{0}\right)$ families of functions $S_{s d} \in C^{\infty}(\widetilde{\mathbb{A}}), H_{s d} \in C^{\infty}\left(\mathbb{D}^{2}\right)$ such that $\widetilde{S}_{s d}=0$ outside $\widetilde{\mathbb{A}}_{d}$ and

$$
\partial_{\xi}^{q}\left(H_{s}^{\prime}\left(y, \xi-\nabla_{y} \widetilde{S}_{s d}(y, \xi)\right)-H_{s d}(\xi)\right)=0, \quad 0 \leq q \leq 1
$$

on $\mathbb{T}^{2} \times \widetilde{E}_{s d}$, where

$$
\widetilde{E}_{s d}=\left\{\xi \in \mathbb{D}_{d}: \nabla_{\xi} H_{s d}(\xi) / 2 \pi \in \Theta^{d}\right\}
$$

(ii) for any $\beta \geq \alpha$,

$$
\left\|\widetilde{S}_{s d}\right\|_{\widetilde{\beta}, \widetilde{\mathbb{A}}_{d} ; \gamma_{d}} \leq C_{\beta} \gamma_{d}^{-1} d^{-(\beta+1) / 2}\left\|H_{s}^{\prime}-H_{s}^{0}\right\|_{\tilde{p}}
$$

where $\tilde{p}=\beta \lambda+\lambda+\sigma$ and $\widetilde{\beta}=\beta-(\lambda-\sigma) / \lambda$ is not in $\Lambda$.

The proof of Theorem A.2 is similar to that of Theorem A in [19] (see also Theorem 5.4 and the Appendix in [13]) and we omit it. The continuity of $\widetilde{S}_{s d}$ and $H_{s d}$ with respect to $s \in U\left(s_{0}\right)$ in the corresponding $C^{\infty}$ spaces follows from the arguments in A.2, Appendix, in [21].

Consider the pair $H_{s}^{0}, H_{s}^{\prime}$ defined above. Obviously, $H_{s}^{0}$ satisfies (A.7) if $c$ is sufficiently small. Fix $\lambda>\sigma+1$ and $\beta>0$ such that $\widetilde{\beta}=\beta-(\lambda-\sigma) / \lambda$ is not in $\Lambda$ and $N \leq \beta \leq N+1$. Set $p=\beta \lambda+\lambda+\sigma$. Lemma A.2 implies

$$
\left\|H_{s}^{\prime}-H_{s}^{0}\right\|_{p, \widetilde{\mathbb{A}}_{d} ; \gamma_{d}} \leq C_{N} a^{M_{1}} \leq \varepsilon \gamma_{d}^{N+2} a^{2 N+4}, \quad M_{1}=N^{2}+4 N+5,
$$

if $d=a^{2}$ and

$$
0<a \leq \varepsilon_{N} \mu^{M}, \quad M=N+2, \quad \varepsilon_{N}=\varepsilon / C_{N} .
$$

Hence, we can apply Theorem A.2. The corresponding function $\widetilde{S}_{s d}$ satisfies the estimate

$$
\left\|\widetilde{S}_{s d}\right\|_{\widetilde{\beta}, \widetilde{\mathbb{A}}_{d} ; \gamma_{d}} \leq C_{N}^{\prime} \gamma_{d}^{N+1} a^{N+1}
$$

which implies

$$
\left|\partial_{\theta}^{p} \partial_{\xi}^{q} \widetilde{S}_{s d}(\theta, \xi)\right| \leq C_{p, N}^{\prime \prime} a^{N}, \quad \text { for } 0<a \leq \varepsilon(\mu, N), \quad|q| \leq N
$$

where $C_{p, N}^{\prime \prime}$ do not depend on $\mu$ and $a$. Moreover, $\widetilde{S}_{s d}=0$ outside a neighborhood of $\mathbb{T} \times \mathbb{D}_{d}$ in $\widetilde{\mathbb{A}}_{d}$ and it generates an exact symplectic transformation taking $\varepsilon_{N}$ smaller if necessary. As in the Appendix, [21] we complete the proof of Theorem A.1.

We are going to patch together the exact symplectic mappings $\chi_{s a}$. Fix $N_{j} \in \mathbb{Z}_{+}$, $\mu_{j}>0$ and $a_{j} \in\left(0, \varepsilon\left(\mu_{j}, N_{j}\right)\right), j=1,2$, and consider the corresponding functions $K_{s j}$ and $S_{s j}$ given by Theorem A.1. Let $\chi_{s j}$ be the exact symplectic mapping with a generating function $S_{s \jmath}$. Denote by $\mathscr{J}_{s j}(\omega)$ the inverse to the frequency mapping $\Gamma \ni I \rightarrow \tau_{s j}^{\prime}(I) / 2 \pi$. 
Proposition A.1. We have

$$
\begin{gathered}
\mathscr{J}_{s 1}(\omega)=\mathscr{Z}_{s 2}(\omega), \quad K_{s 1}\left(\mathscr{J}_{s 1}(\omega)\right)=K_{s 2}\left(\mathscr{J}_{s 1}(\omega)\right), \\
\chi_{s 1}\left(\theta, \mathscr{J}_{s 1}(\omega)\right)=\chi_{s 2}\left(\theta+r\left(\mathscr{J}_{s 1}(\omega)\right), \mathscr{J}_{s 1}(\omega)\right),
\end{gathered}
$$

for any $\omega \in \Theta^{1,2}:=\Theta^{0}\left(a_{1}, \mu_{1}, N_{1}\right) \cap \Theta^{0}\left(a_{2}, \mu_{2}, N_{2}\right)$, where $r_{s j}(I)=S_{s 1}(0, I)$ $S_{s 2}(0, I)$.

Proof. The proof is close to that of Proposition A.5 in [21]. To simplify the notations we drop the index $s$. Suppose that $\Theta^{1,2}$ is not empty. Set $B_{j}=\chi_{j}^{-1} \circ B \circ \chi_{j}$ and consider $\psi=\chi_{1}^{-1} \circ \chi_{2}$. Then $B_{2}=\psi^{-1} \circ B_{1} \circ \psi$ and (4.2) implies

$$
B_{j}^{*} \sigma_{0}-\sigma_{0}=d f_{j}, \quad \sigma_{0}=I d \varphi, \quad j=1,2
$$

where

$$
f_{j}\left(\varphi, \mathscr{J}_{j}(\omega)\right)=2 \pi \mathscr{T}_{j}(\omega), \quad \omega \in \Theta^{0}\left(a_{j}, \mu_{j}, N_{j}\right), \quad j=1,2,
$$

according to (4.3). On the other hand,

$$
\psi^{*} \sigma_{0}-\sigma_{0}=d \phi
$$

where $\phi$ is a smooth function in $\mathbb{A}$. As in the proof of (4.6) we deduce from (A.12) and (A.14) that

$$
f_{2}(\varphi, I)=f_{1}(\psi(\varphi, I))+\phi(\varphi, I)-\phi\left(B_{2}(\varphi, I)\right)+C, \quad(\varphi, I) \in \mathbb{A},
$$

where $C=0$. As the invariant circles $\Lambda(\omega)$ are uniquely determined by their rotation numbers $\omega \in \Theta$, we have

$$
\psi\left(\mathbb{T} \times \mathscr{F}_{2}(\omega)\right)=\mathbb{T} \times \mathscr{J}_{1}(\omega), \quad \omega \in \Theta^{1,2} .
$$

Using (A.13) and (A.16) we obtain

$$
2 \pi \mathscr{T}_{2}(\omega)=2 \pi \mathscr{T}_{1}(\omega)+\phi\left(\varphi, \mathscr{J}_{2}(\omega)\right)-\phi\left(\varphi+\omega, \mathscr{J}_{2}(\omega)\right), \quad(\varphi, \omega) \in \mathbb{T} \times \Theta^{1,2},
$$

which implies

$$
2 \pi \mathscr{T}_{2}(\omega)=2 \pi \mathscr{T}_{1}(\omega), \quad \omega \in \Theta^{1,2} .
$$

Since $\Theta^{1,2}$ has no isolated points, differentiating the last equality with respect to $\omega$ we prove (A.10).

According to (A.10) and (A.16) we have

$$
\psi\left(\mathbb{T} \times \mathscr{J}_{2}(\omega)\right)=\mathbb{T} \times \mathscr{J}_{2}(\omega), \quad \omega \in \Theta^{1,2,},
$$

and we obtain

$$
\nabla_{\theta} S_{1}\left(\theta, \mathscr{J}_{2}(\omega)\right)=\nabla_{\theta} S_{2}\left(\theta, \mathscr{J}_{2}(\omega)\right), \quad(\theta, \omega) \in \mathbb{T} \times \Theta^{1,2} .
$$

This proves (A.11).

Proof of Theorem 3.1. First we fix $N$ in $\mathbb{Z}_{+}$. Take $a_{0}=\varepsilon(\mu, N), a_{j}=a_{0} C_{0}^{j}$, $\gamma_{\jmath}=\mu_{0} a_{j}^{N}, 0<\mu_{0}<\mu$, and denote by $K_{s j}(I)$ and $S_{s j}(\theta, I)$ the corresponding functions given by Theorem A.1 for $a=a_{j}, j=1,2, \ldots$ Replacing $S_{s j}(\theta, I)$ by $S_{s j}(\theta, I)-S_{s j}(0, I)$ we can suppose that $S_{s j}(0, I)=0$. Using Proposition A.1, (A.3) and (A.4), and applying a suitable Whitney extension theorem we find smooth 
functions $\mathscr{J}_{s}^{N}(\omega), K_{s}^{N}(I)$ and $S_{s}^{N}(\theta, I)$ in $\mathbb{R}$ and $\mathbb{T} \times \mathbb{R}$ respectively which depend continuously on $s$ and such that the equalities

$$
\begin{gathered}
\mathscr{J}_{s}^{N}(\omega)=\mathscr{J}_{s j}(\omega), \quad K_{s}^{N}\left(\mathscr{J}_{s}^{N}(\omega)\right)=K_{s j}\left(\mathscr{J}_{s}^{N}(\omega)\right) \\
S_{s}^{N}\left(\theta, \mathscr{J}_{s}^{N}(\omega)\right)=S_{s j}\left(\theta, \mathscr{J}_{s}^{N}(\omega)\right)
\end{gathered}
$$

as well as their derivatives with respect to $(\theta, \omega)$ are satisfied in $\mathbb{A}^{j} \equiv \mathbb{T} \times$ $\Theta^{0}\left(a_{\jmath}, \mu_{0}, N\right)$. Denote by $\chi_{s}^{N}$ the exact symplectic mapping generated by $S_{s}^{N}$ and by $(\varphi, I)$ the corresponding symplectic coordinates in $\mathbb{T} \times \mathbb{R}$. Then

$$
B_{s}^{0}=\left(\chi_{s}^{N}\right)^{-1} \circ B_{s} \circ \chi_{s}^{N}
$$

is generated by

$$
\tau_{s}^{N}(I)+Q_{s}^{N}(\varphi, I), \quad \tau_{s}^{N}(I)=-\frac{4}{3} K_{s}^{N}(I)^{3 / 2},
$$

where $Q_{s}^{N}(\varphi, I)$ is a smooth function and $Q_{s}^{N}\left(\varphi, \mathscr{J}_{s}^{N}(\omega)\right)$ has a zero of infinite order on each $\mathbb{A}^{j}, j=1,2, \ldots$. Fix $\mu_{0}=\mu C_{0}^{N}$ and take $a \in\left[a_{\jmath}, a_{j-1}\right], d=a^{2}$. Then $\gamma_{\jmath}<\gamma_{\jmath-1} \leq \gamma_{d}$ and the Cantor set $\Theta^{*}(a, \mu, N)$ is contained in the union of the sets $\mathbb{A}^{j-1}$ and $\mathbb{A}^{j}$. Hence, $Q^{0}\left(\varphi, \mathscr{J}_{s}^{N}(\omega)\right)$ has a zero of infinite order on

$$
\Theta_{N}=\cup\left\{\Theta^{*}(a, \mu, N): 0<a \leq \varepsilon(\mu, N)\right\}
$$

Moreover, $K_{s}^{N}, Q_{s}^{N}$ and $S_{s}^{N}$ satisfy (A.3) and (A.4). Take integers $N_{2}>N_{1} \geq 1$, pick $a$ in $\left(0, \varepsilon\left(\mu, N_{2}\right)\right]$, and set $\gamma_{j}=\mu a^{N_{j}}$. Then $\gamma_{2}<\gamma_{1}$ and we obtain

$$
\Theta_{N_{1}} \cap\left(0, \varepsilon\left(\mu, N_{2}\right)\right] \subset \Theta_{N_{2}}
$$

Using Proposition A.1 as well as (A.3) and (A.4) we obtain smooth (in the sense of Whitney) functions $\mathscr{J}_{s}(\omega), K_{s}\left(\mathscr{J}_{s}(\omega)\right)$ and $S_{s}\left(\theta, \mathscr{J}_{s}(\omega)\right)$ in $\Theta$ and $\mathbb{T} \times \Theta$ respectively such that

$$
\begin{gathered}
\mathscr{Z}_{s}(\omega)=\mathscr{J}_{s}^{N}(\omega), \quad K_{s}\left(\mathscr{J}_{s}(\omega)\right)=K_{s}^{N}\left(\mathscr{Z}_{s}(\omega)\right) \\
S_{s}\left(\theta, \mathscr{J}_{s}(\omega)\right)=S_{s}^{N}\left(\theta, \mathscr{J}_{s}(\omega)\right)
\end{gathered}
$$

for any $(\theta, \omega) \in \mathbb{T} \times \Theta$. Denote by $\widetilde{S}_{s}(\theta, \omega)$ and $\widetilde{\mathscr{J}}_{s}(\omega)$ suitable smooth Whitney extensions of $\widetilde{S}_{s}\left(\theta, \mathscr{J}_{s}(\omega)\right),(\theta, \omega) \in \mathbb{T} \times \Theta$ and $\mathscr{J}_{s}(\omega), \omega \in \Theta$, which depend continuously on $s$. Let $\widetilde{K}_{s}^{\prime}(I)$ be the function inverse to $\omega \rightarrow \mathscr{J}_{s}(\omega)$. Taking $\chi_{s}$ to be the exact symplectic mapping generated by $S_{s}(\theta, I)=\widetilde{S}_{s}\left(\theta, \widetilde{K}_{s}^{\prime}(I)\right)$ and using (A.3) and (A.4) we complete the proof of Theorem 3.1.

Acknowledgements. This paper has been written under the support of the Alexander von Humboldt foundation. The main part of it was prepared during my stay in TH Darmstadt and I would like to thank Hans-Dieter Alber for the hospitality and the stimulating discussions. I would like to thank Jürgen Pöschel for the helpful discussions about KAM theory. 


\section{References}

1. Amiran, E.: Caustics and evolutes for convex planar domains. J. Diff. Geom. 28, 2, 345-358 (1988)

2. Anderson, K., Melrose, R.: The propagation of singularities along gliding rays. Invent. Math. 41, 197-232 (1977)

3. Cardoso, F., Popov, G.: Rayleigh quasimodes in linear elasticity. Commun. Partial Differ. Equations 17, 1327-1367 (1992)

4. Colin de Verdière, Y.: Sur les longueurs des trajectoires périodiques d'un billiard. In: Dazord, P., Desolneux-Moulis, N. (eds.) Géométrie Symplectique et de Contact: Autour du Théorème de Poincaré-Birkhoff. Travaux en Cours, Sém. Sud-Rhodanien de Géométrie III, Paris: Herman 1984, pp. 122-139

5. Colin de Verdière, Y.: Quasi-modes sur les varietes Riemanniennnes. Invent. Math. 43, 15-52 (1977)

6. Douady, R.: Une démonstration directe de l'équivalence des théorèmes de tores invariants pour difféomorphismes et champs de vecteurs. C. R. Acad. Sci., Paris, Ser. A. 295, 201-204 (1982)

7. Duistermaat, J.: Oscillatory integrals, Lagrange immersions and infolding of singularities. Commun. Pure Appl. Math. 27, 207-281 (1974)

8. Fransçoise, J.P., Guillemin, V.: On the period spectrum of a symplectic map. J. Funct. Anal. 100, 317-358 (1991)

9. Guillemin, V., Melrose, R.: A cohomological invariant of discrete dynamical systems. In: Putzer, P., Feher, F. (eds.): Christoffel Centennial Volume, Basel: Birkhäuser 1981, pp. 672-679

10. Guillemin, V., Melrose, R.: The Poisson summation formula for manifolds with boundary. Adv. Math. 32, 204-232 (1979)

11. Hörmander, L.: The analysis of linear partial differential operators. III, IV. Berlin, Heidelberg, New York: Springer 1985

12. Kac, M.: Can one hear the shape of a drum. Am. Math. Soc. Monthly. 73, 4, Part II, 1-23 (1966)

13. Kovachev, V., Popov, G.: Invariant tori for the billiard ball map. Trans. Am. Math. Soc. 317, 45-81 (1990)

14. Lazutkin, V.: The existence of caustics for a billiard problem in a convex domain. Math. USSR Izv. 7, 185-214 (1973)

15. de la Llave, R., Marco, J., Moriyón, R.: Canonical perturbation of Anosov systems and regularity results for the Livsic cohomology equations. Ann. Math. 123, 537-611 (1986)

16. Marvizi, Sh., Melrose, R.: Spectral invariants of convex planar regions. J. Differ. Geom. 17, 475-502 (1982)

17. Moser, J.: On invariant curves of area preserving mappings of an annulus. Nachr. Akad. Wiss. Götingen Math. Phys. 1-20 (1962)

18. Petkov, V., Stojanov, L.: Geometry of reflecting rays and inverse spectral results. New York: Wiley 1992

19. Pöschel, J.: Integrability of Hamiltonian systems on Cantor sets. Commun. Pure Appl. Math. 35, 653-696 (1982)

20. Popov, G.: Glancing hypersurfaces and length spectrum invariants, Preprint (1990)

21. Popov, G.: Length spectrum invariants of Riemannian manifolds. Math. Z. 213, 311-351 (1993)

22. Soga, H.: Oscillatory integrals with degenerate stationary points and their application to the scattering theory. Commun. Partial Differ. Equations 6, 273-287 (1981) 